\title{
Optimal Trading Mechanisms with Ex Ante Unidentified Traders*
}

\author{
$\mathrm{Hu} \mathrm{Lu}$ \\ Department of Economics, University of Ottawa \\ and \\ Jacques Robert \\ Department of Economics, University of Montreal
}

This version, January 2000

\footnotetext{
${ }^{*}$ We would like to thank the Associate Editor and a referee for very helpful suggestions. The financial support from SSHRCC and FCAR is gratefully acknowledged. We also thank Paul Johnson and Bernard Lebrun for helpful comments. All errors are our responsibility.
} 
ABSTRACT: We analyze optimal trading mechanisms in an exchange economy where each trader owns some units of a good to be traded and may be either a seller or a buyer, depending on the realization of the privately observed valuations. The concept of virtual valuation is extended to ex ante unidentified traders; contrary to the case where each trader is assigned a role as either a buyer or a seller, the traders' virtual valuations now depend on the choice of the trading mechanism and are generally non-monotonic even if the distribution of valuations is regular. We show that the trading mechanisms that maximize a broker's expected profit or expected total gains from trade are generalized double auctions which maximize the gains from trade measured in some modified monotonic virtual valuations for the traders. The bunching phenomena, which are here specific to ex ante unidentified traders, will be a general feature in these mechanisms. Furthermore, the randomization rule by which ties are broken is now part of the design of the optimal mechanisms. Finally, we show that the optimal mechanism converges toward a simple bid-ask mechanism as the number of participants in the market increases.

KEYWORDS: Mechanism design, efficient trading, intermediation, double auctions, ask-bid mechanism.

JEL Classification Numbers: D44, D82. 


\section{Introduction}

Myerson and Satterthwaite [15] consider bargaining problems between one buyer and one seller for a single object. They show that the buyer and seller are unable to exhaust gains from trade if they have incomplete information about each other and there is positive probability that there are no gains from trade. They also show how to compute mechanisms that maximize expected total gains from trade, and mechanisms that maximize a broker's expected profit. Since Myerson and Satterthwaite's work, the literature has explored the implementability of ex post efficient allocations and mechanisms which are efficient in some sense other than ex post. ${ }^{1}$ With some exceptions, it is assumed in the literature, as in Myerson and Satterthwaite, that traders are ex ante identified buyers or sellers; that is, either a seller sells a buyer a single unit, or no trade occurs. In Cramton, Gibbons, and Klemperer [2], McAfee [11], and Spulber [19], this assumption is relaxed. Spulber [19] considers interim efficient mechanisms, McAfee [11] examines ex post efficiency with continuous quantities, and Cramton et al. [2] investigate efficient mechanisms for dissolving a partnership.

This paper considers the problem of designing a rule to determine the terms of trade among several traders who own some units of a good to be traded and have private information about their preferences. In this context, a trader holding some units of the good (but less than his satiated demand level) may be either a seller or a buyer, depending on the realization of the privately observed information and the choice of the mechanism; his role as a buyer or a seller is endogenously determined by the traders' bids, but cannot be identified prior to trade. The multilateral trading problem studied here extends models of Myerson [13], Myerson and Satterthwaite [15], and Gresik and Satterthwaite [3] in which one unit of the good is demanded or supplied inelastically by each trader, and the model of Cramton et al. [2] in which traders have the highest level of demand possible (the economy wide endowment). The fact that a trader may be on either side of a trade creates difficulties beyond those of the standard mechansim design problems and has important implications for the nature of the optimal mechanisms.

Myerson and Satterthwaite [15] show that with incomplete information ex post efficiency cannot be achieved when the asset is owned by a single party. In contrast, Cramton, Gibbons, and Klemperer

\footnotetext{
${ }^{1}$ These issues have been the focus of a number of papers including Gresik and Satterthwaite [3], Leininger et al. [7], Makowski and Mezzetti [9], Rustichini et al. [17], Satterthwaite and Williams [18], Williams [20], and Wilson [21].
} 
[2] show that if the ownership is distributed among a partnership, ex post efficient allocation is possible provided no single partner owns too large a share. McAfee [11] reinforces the conclusion of Cramton et al. [2] by considering the hidden endowments model which symmetrizes the agents. Lu [8] gives an explanation to these contrasting results by considering different preferences. He shows that the implementability of efficient allocations is primarily determined by the degree of revelation of private information required in achieving it. If the achievement of ex post efficiency requires full revelation of private information, it may be impossible to achieve ex post efficiency when traders are not ex ante identical. If the traders have inelastic demands for a fixed number of units, partial revelation of private information is sufficient to allocate the goods efficiently, and this allows for ex post efficiency to be possible for sufficiently symmetric distributions. Thus, the results of Myerson and Satterthwaite and Cramton et al. are two extreme cases.

This paper characterizes the revenue-maximizing mechanism and the ex ante efficient mechanism (in the sense of Holmström and Myerson [4]) when the traders have inelastic demands for a fixed number of units. In the standard mechansim design literature (Myerson and Satterthwaite [15], and many other similar papers), the minimum utility is always achieved by the highest or lowest type independently of the choice of the mechanism, and the continuum of individual rationality constraints are reduced to those for the highest or lowest types that are binding at the optimum. As a result, the only constraints are the monotonicity of the allocations, which can also be ignored by assuming that the distribution of types is regular, and optimal allocations are given by pointwise solutions. In the present model, since it is no longer clear who is selling and who is buying prior to revelation of types, traders with high types typically expect to be buyers, those with low types expect to be sellers, and those in the middle do not expect to trade. Thus, contrary to the standard cases, the minimum utility types for ex ante unidentified traders are those in the middle and generally depend on the choice of the trading mechanism. Moreover, the monotonicity of a trader's virtual valuations fails even if the distribution of types is regular, since high types expect to be buyers who have an incentive to understate their valuations and low types expect to be sellers who tend to overstate their valuations. Thus we must consistently determine the traders' worst-off types who expect to be neither a buyer nor a seller as well as the allocation rules and, at the same time, maximize the objective function. 
The revenue-maximizing mechanism and the ex ante efficient mechanism are characterized by solving the trading mechanism that maximizes a weighted sum of expected total gains from trade and expected profit to the market maker. The optimal trading mechanism is characterized by some modified monotonic virtual valuations: the goods will be assigned to the traders whose modified virtual valuations are highest and ties will be broken by randomizing. In the optimal mechanism, the participation constraints will be binding for some types other than the highest and lowest types, and the bunching phenomenon which here is specific to ex ante unidentified traders will be a general feature. An algorithm is provided to compute the ranges of bunching that are uniquely determined by the traders' initial endowments. The characterization of the optimal trading mechanism must also include a randomization rule by which ties are broken. Unlike the standard models where bunching is due to the irregularity of the distribution of valuations and all randomization rule is valid to break ties, now the tie breaking rule is strictly restricted: it must make types in the middle to have expected net trade zero. Hence the randomization rule becomes an important instrument in the design of the optimal mechanism. Such rules can be constructed by an algorithm.

Finally, we consider what happens to the optimal mechanisms when the market becomes large. We show that they converge towards simple bid-ask price mechanisms. Bid-ask price mechanisms are common in many trading institutions, in particular financial market conducted by market makers. The paper provides some theoretical justification for these institutions.

The rest of the paper is organized as follows. In section 2, we first define the formal structure of the multilateral trading problem. We then present a general characterization of all incentive compatible and individually rational mechanisms. In section 3, we show how to construct the trading mechanism that maximizes a weighted sum of expected total gains from trade and expected profit to the market maker. In section 4, we show that the ex ante efficient mechanism can be characterized by the optimal mechanism obtained in section 3 for some weight. In section 5 , we consider where the number of agents in the economy becomes large, we show that the optimal mechanism converges to a ask-bid mechanism. 


\section{An Exchange Economy with Ex Ante Unidentified Traders}

We consider an exchange economy composed of $n$ traders indexed by $i \in N=\{1,2, \cdots, n\}$. Each trader $i$ owns $k_{i}$ units of an indivisible good to be traded and is privately informed about a preference parameter (his "type") $v_{i}$. Other traders do not observe a trader's type $v_{i}$, but it is common knowledge that the types are drawn independently from a distribution $F$ with support $[\underline{v}, \bar{v}]$ and positive continuous density $f$. Throughout, we shall assume that the traders want to hold at most $k_{0}$ units of the good and $k_{i} \leq k_{0}$ for all $i \in N$; that is, no trader is initially endowed with more than what he wants to hold. The fact that traders all have some endowments means that any trader may be a buyer or a seller in the trading game, and trader $i$ has an inelastic demand for $k_{0}-k_{i}$ units or supply of $k_{i}$ units when he is a buyer or seller.

A trader with type $v_{i}$ and initial endowment $k_{i}$ has preferences represented by the utility function

$$
u_{i}\left(q, t, v_{i}\right)=v_{i} \min \left(q, k_{0}-k_{i}\right)-t,
$$

where $q \geq-k_{i}$ is the net number of units bought by the trader and $t$ is total spending on these units. Each trader's utility function is normalized so that if he is to neither trade units nor make or receive a cash payment, then his utility is zero for all type $v_{i} \cdot{ }^{2}$ Note that $v_{i}$ is the trader's reservation price or valuation for each of the $k_{0}$ first units of the good. ${ }^{3}$

The traders are going to participate in some trading mechanism to determine, first, who will purchase additional units and who will sell their initial endowments and, second, how much a buyer should pay for the units he bought and how much a seller should be paid for the units he sold. Our general question is: what kinds of trading mechanisms can be designed that have good economic efficiency properties?

We consider the direct revelation mechanisms in which traders simultaneously report their valuations ${ }^{4}$ $v=\left(v_{1}, v_{2}, \cdots, v_{n}\right)$ to a coordinater or market-maker who then determines an allocation $q(v)=$

\footnotetext{
${ }^{2}$ By normalizing the utility function, the reservation utility $u_{i}\left(0,0, v_{i}\right)=0$ is brought to be independent of type, but we will see, not as generally assumed in the literature, that the expected utility is not monotonically increasing with the type. If the utility is expressed in terms of gross surplus, the reservation utility is then type dependent.

${ }^{3} \mathrm{An}$ alternative assumption is that each trader has a vector of valuations $v_{i}=\left(v_{i}^{1}, v_{i}^{2}, \cdots, v_{i}^{k_{0}}\right)$, where $v_{i}^{j}$ represents the trader's valuation of his $j^{\text {th }}$ unit of the good. If these valuations are not perfectly correlated, it involves a problem of multidimensional uncertainty which appears to be much more complicated. (see Laffont, Maskin, and Rochet [6], and Rochet [16]) For simplicity, we restrict ourselves to the one-dimensional case.

${ }^{4}$ We assume that the initial endowments $\left(k_{1}, k_{2}, \ldots, k_{n}\right)$ are common knowledge, but it is not essential. If the total number of units of the good $K=\sum_{i=1}^{n} k_{i}$ is known, we can ask the traders to report their numbers of units and then forbid trade if the total number reported does not equal $K$, and implement the mechanism if the reports agree with $K$.
} 
$\left(q_{1}(v), \cdots, q_{n}(v)\right)$ and a payment $t(v)=\left(t_{1}(v), \cdots, t_{n}(v)\right)$, where $q_{i}$ is the net trade for trader $i$ and $t_{i}$ is the net money transfer from trader $i$. We require that these allocations balance: $\sum_{i=1}^{n} q_{i}(v)=0$ for all $v \in[\underline{v}, \bar{v}]^{n}$. For the moment, no restriction is made on $\sum_{i=1}^{n} t_{i}(v)$, but the budget balance condition will be required in section 4 when we look for ex ante efficient mechanisms. Since all traders want to hold at most $k_{0}$ units, we can assume that $-k_{i} \leq q_{i}(v) \leq k_{0}-k_{i}$ for all $v \in[\underline{v}, \bar{v}]^{n}$ and $i \in N$. Also, we assume that each trader is endowed with enough money that any required transfer is feasible. The pair of outcome functions $\{q, t\}$ is referred to as a direct trading mechanism.

Let $-i=N \backslash\{i\}$ and let $E_{-i}[\cdot]$ be the expectation operator with respect to $v_{-i}$. Then $Q_{i}\left(v_{i}\right)=$ $E_{-i}\left[q_{i}\left(v_{i}, v_{-i}\right)\right]$ is $i$ 's expected net trade, and $T_{i}\left(v_{i}\right)=E_{-i}\left[t_{i}\left(v_{i}, v_{-i}\right)\right]$ is $i$ 's expected payment when he announces $v_{i}$. Consequently, the trader's expected payoff is

$$
U_{i}\left(v_{i}\right)=E_{-i}\left[u_{i}\left(q_{i}(v), t_{i}(v), v_{i}\right)\right]=v_{i} Q_{i}\left(v_{i}\right)-T_{i}\left(v_{i}\right)
$$

The trading mechanism $\{q, t\}$ is incentive compatible if each type of each trader wants to report his private information truthfully when others report truthfully:

$$
U_{i}\left(v_{i}\right) \geq v_{i} Q_{i}\left(\hat{v}_{i}\right)-T_{i}\left(\hat{v}_{i}\right), \quad \forall i \in N \quad \forall v_{i}, \hat{v}_{i} \in[\underline{v}, \bar{v}] .
$$

By the Revelation Principle (Myerson [13], among others), there is no loss of generality in restricting our attention to incentive compatible direct mechanisms. The mechanism $\{q, t\}$ is interim individually rational if all types of all traders are better off participating in the mechanism (in terms of their expected payoff) than holding their initial endowments:

$$
U_{i}\left(v_{i}\right) \geq 0 \quad \forall i \in N \text { and } v_{i} \in[\underline{v}, \bar{v}] .
$$

The mechanism $\{q, t\}$ is incentive feasible if it is incentive compatible and individually rational. We will also say that an allocation $q$ is implementable if there exists a transfer function $t$ such that $\{q, t\}$ is an incentive feasible mechanism. The following lemma develops a necessary and sufficient condition for a mechanism to be incentive feasible. The proof is in the Appendix.

Lemma 1. A trading mechanism $\{q, t\}$ is incentive feasible if and only if for every $i \in N, Q_{i}\left(v_{i}\right)$ is non-decreasing and

$$
U_{i}\left(v_{i}\right)=U_{i}\left(v_{i}^{*}\right)+\int_{v_{i}^{*}}^{v_{i}} Q_{i}(u) d u \quad \forall v_{i} \in[\underline{v}, \bar{v}]
$$




$$
U_{i}\left(v_{i}^{*}\right) \geq 0
$$

where ${ }^{5}$

$$
v_{i}^{*} \in V^{*}\left(Q_{i}\right)=\left\{v_{i} \mid Q_{i}(u) \leq 0, \forall u<v_{i} ; Q_{i}(w) \geq 0, \forall w>v_{i}\right\}
$$

For any allocation $q=\left(q_{1}, \cdots, q_{n}\right)$ such that $Q_{i}\left(v_{i}\right)$ is non-decreasing for all $i \in N, V^{*}\left(Q_{i}\right)$ is well-defined in (5) and will be called the worst-off types of trader $i$ under allocation $q$. Equation (3) implies that expected net utility $U_{i}\left(v_{i}\right)$ is continuous and convex in $v_{i}$. Moreover, from (3) and (5), $U_{i}\left(v_{i}\right)$ is minimized at any $v_{i}^{*} \in V^{*}\left(Q_{i}\right)$, so the continuum of constraints in (2) can be reduced to the individual rationality constraint for a single $v_{i}^{*}$ in $(4)$. It is easy to show that $V^{*}\left(Q_{i}\right)$ is a singleton or a closed interval and all worst-off types in the interior of $V^{*}\left(Q_{i}\right)$ satisfy $Q_{i}\left(v_{i}^{*}\right)=0$. Intuitively, as in Cramton et al. [2], a worst-off type expects on average to be neither a buyer nor a seller of the good, and therefore he has no incentive to overstate or understate his valuation. Hence, he does not need to be compensated in order to induce him to report his valuation truthfully. In general, given an incentive feasible mechanism $\{q, t\}$, it is no longer clear who is selling and who is buying prior to revelation of types, but on average trader $i$ is a buyer if his type $v_{i} \geq \max V^{*}\left(Q_{i}\right)$ and a seller if his type $v_{i} \leq \min V^{*}\left(Q_{i}\right)$.

Let us define, for any $\lambda \geq 0$ and $v \in[\underline{v}, \bar{v}]$

$$
\alpha(v \mid \lambda)=v-\lambda \frac{1-F(v)}{f(v)} \quad \text { and } \quad \beta(v \mid \lambda)=v+\lambda \frac{F(v)}{f(v)} .
$$

$\alpha(v \mid \lambda)$ and $\beta(v \mid \lambda)$ are referred to as the $\lambda$-virtual valuation of "buyer-type" and "seller-type", respectively. Given an incentive feasible mechanism $\{q, t\}$, for any $v_{i}^{*} \in V^{*}\left(Q_{i}\right)$, let

$$
\eta\left(v_{i} \mid v_{i}^{*}, \lambda\right)= \begin{cases}\beta\left(v_{i} \mid \lambda\right), & \text { if } v_{i}<v_{i}^{*}, \\ v_{i}^{*}, & \text { if } v_{i}=v_{i}^{*} \\ \alpha\left(v_{i} \mid \lambda\right), & \text { if } v_{i}>v_{i}^{*}\end{cases}
$$

$\eta\left(v_{i} \mid v_{i}^{*}, \lambda\right)$ is referred to as a $\lambda$-virtual valuation under allocation $q \cdot{ }^{6}$ Virtual valuations will play a crucial role in construction of optimal trading mechanisms.

\footnotetext{
${ }^{5}$ This lemma can be seen as a combination of Lemmas 1-3 of Cramton et al. [2] except that we redefine $V^{*}\left(Q_{i}\right)$ by nonstrict inequalities. $V^{*}\left(Q_{i}\right)$ is now well-defined for all non-decreasing function $Q_{i}$. If $V^{*}\left(Q_{i}\right)$ was as defined in Lemma 2 of Cramton et al. by strict inequalities, $V^{*}\left(Q_{i}\right)$ would be empty when $Q_{i}$ is constantly zero on an interval. Since Cramton et al. only investigate the possibility of ex post efficiency and the expected net trade $Q_{i}\left(v_{i}\right)$ is strictly increasing in an ex post efficient allocation, $V^{*}\left(Q_{i}\right)$ is a singleton in their paper. But we look for optimal trading mechanisms that may have $Q_{i}$ constant on an interval (as will be seen, actually a positive mass of types in the middle expect to be neither a buyer nor a seller in these mechanisms), so the definition must be corrected so that $V^{*}\left(Q_{i}\right)$ is meaningful for all implementable allocations.

${ }^{6}$ Myerson [14] introduced the concept of virtual valuation for ex ante identified traders. We extend this concept to ex ante unidentified traders.
} 
Before proceeding further we should discuss virtual valuations. As observed earlier, a trader's worst-off types $V^{*}\left(Q_{i}\right)$ typically are between $\underline{v}$ and $\bar{v}$, then his virtual valuations are of both "buyertype" and "seller-type". If $\lambda=0$, then $\eta\left(v_{i} \mid v_{i}^{*}, 0\right)=v_{i}$. The virtual valuations equal the true valuations under any implementable allocation. If, however, $\lambda>0$, then $\alpha\left(v_{i} \mid \lambda\right)<v_{i}<\beta\left(v_{i} \mid \lambda\right)$ for all $v_{i} \in(\underline{v}, \bar{v})$. Thus, for $\lambda>0$, a trader's virtual valuations are distorted downward (upward) to be below (above) his true valuations when he expects to be a buyer (seller). Intuitively, these distortions express the strategic behavior that buyers have an incentive to understate their valuations and sellers tend to overstate their valuations. Moreover, since $\alpha\left(v_{i} \mid \lambda\right)<v_{i}<\beta\left(v_{i} \mid \lambda\right)$ when $\lambda>0, \eta\left(v_{i} \mid v_{i}^{*}, \lambda\right)$ is discontinuous at $v_{i}=v_{i}^{*}$ and is not monotonically increasing in $v_{i}$ over $[\underline{v}, \bar{v}]$ for all distribution of valuations. If $\alpha\left(v_{i} \mid 1\right)$ and $\beta\left(v_{i} \mid 1\right)$ are both strictly increasing, it is straightforward to verify that for every $\lambda \in[0,1], \alpha\left(v_{i} \mid \lambda\right)$ and $\beta\left(v_{i} \mid \lambda\right)$ are also strictly increasing, so $\eta\left(v_{i} \mid v_{i}^{*}, \lambda\right)$ is increasing over $\left[\underline{v}, v_{i}^{*}\right)$ and $\left(v_{i}^{*}, \bar{v}\right]$ but there is a "buyer-seller" spread at $v_{i}^{*}$.

The following lemma characterizes implementable allocations and expresses the expected revenue from incentive feasible mechanisms solely in terms of the allocation rule. The proof is in the Appendix.

Lemma 2. For any allocation $q=\left(q_{1}, \cdots, q_{n}\right)$ such that $Q_{i}\left(v_{i}\right)$ is non-decreasing for all $i \in N$, there exists a payment function $t$ such that $\{q, t\}$ is incentive feasible. The maximum expected revenue from any incentive feasible mechanism implementing $q$ is given by

$$
R(q)=E\left[\sum_{i=1}^{n} \eta\left(v_{i} \mid v_{i}^{*}\right) q_{i}(v)\right]=E\left[\sum_{i=1}^{n} \eta\left(v_{i} \mid v_{i}^{*}\right) Q_{i}\left(v_{i}\right)\right]
$$

where $\eta\left(v_{i} \mid v_{i}^{*}\right)=\eta\left(v_{i} \mid v_{i}^{*}, 1\right)$ and $v_{i}^{*} \in V^{*}\left(Q_{i}\right)$ for which the individual rationality is binding, ${ }^{7}$ i.e., $U_{i}\left(v_{i}^{*}\right)=0$.

\section{Optimal Trading Mechanisms}

We now consider the case where the traders are intermediated by a broker (or market-maker) who can be a net source or sink of money, but he cannot himself own the good. First, as in Myerson and Satterthwaite [15], an interesting question is to ask for the mechanism which maximizes the expected

\footnotetext{
${ }^{7}$ Note that when $V^{*}\left(Q_{i}\right)$ is an interval, since the expected net trade $Q_{i}\left(v_{i}\right)$ is zero on this interval, the expected revenue in (7) does not depend on the choice of $v_{i}^{*} \in V_{i}^{*}\left(Q_{i}\right)$.
} 
revenue to the broker, subject to the incentive compatibility and individual rationality constraints. More generally, we can introduce an objective function that is a weighted sum of expected total gains from trade and expected revenues to the market maker, and seek a mechanism that maximizes this objective function subject to the incentive feasibility constraints for traders.

First, we define the objective function. For any $\lambda \in[0,1]$, let

$$
W_{\lambda}(q)=(1-\lambda) E\left[\sum_{i=1}^{n} v_{i} q_{i}(v)\right]+\lambda R(q),
$$

where $R(q)$ is given by (7) of Lemma 2. Our question is to seek a trading mechanism that maximizes $W_{\lambda}(q)$ among all incentive feasible mechanisms. Substituting from $(7), W_{\lambda}(q)$ can be expressed as

$$
W_{\lambda}(q)=E\left[\sum_{i=1}^{n} \eta\left(v_{i} \mid v_{i}^{*}, \lambda\right) q_{i}(v)\right], \text { where } v_{i}^{*} \in V^{*}\left(Q_{i}\right)
$$

Hence, from Lemmas 1 and 2, the maximization problem can be written as

$$
\mathcal{P}_{\lambda}\left\{\begin{array}{l}
\max W_{\lambda}(q)=E\left[\sum_{i=1}^{n} \eta\left(v_{i} \mid v_{i}^{*}, \lambda\right) q_{i}(v)\right] \\
\text { s.t. }-k_{i} \leq q_{i} \leq k_{0}-k_{i} \text { for all } i \text { and } \sum_{i=1}^{n} q_{i}=0 \\
Q_{i}\left(v_{i}\right) \text { is non-decreasing and } v_{i}^{*} \in V^{*}\left(Q_{i}\right) .
\end{array}\right.
$$

Thus solving for the incentive feasible mechanism that maximizes $W_{\lambda}(q)$ boils down to finding the allocation that maximizes the expected total gains from trade measured in $\lambda$-virtual valuations.

Obviously, when $\lambda=1, \mathcal{P}_{1}$ is the maximization of the expected revenue to the broker subject to the incentive feasibility constraints. When $\lambda=0, \mathcal{P}_{0}$ is the maximization of expected total gains from trade and its solution is the ex post efficient allocation, which can be formally defined as ${ }^{8}$

$$
q_{i}^{e}\left(v_{i}, v_{-i}\right)= \begin{cases}k_{0}-k_{i}, & \text { if } v_{i} \text { is among the } n_{0} \text { highest values } \\ r-k_{i}, & \text { if } v_{i} \text { is the }\left(n_{0}+1\right)^{s t} \text { highest value } \\ -k_{i}, & \text { otherwise }\end{cases}
$$

where $n_{0}$ and $r$ are nonnegative integers such that $n_{0} k_{0}+r$ equals the total number of units $\sum_{i=1}^{n} k_{i}$ and $0 \leq r<k_{0}$. In Section 4, we consider more carefully the problem of maximizing expected gains from trade when the budget balance condition is required.

Notice that the virtual valuations $\eta\left(v_{i} \mid v_{i}^{*}, \lambda\right)$ now depend on the allocation rules $q$ through the traders' worst-off types $V^{*}\left(Q_{i}\right)$, which may vary as $q$ vary. Thus, to solve $\mathcal{P}_{\lambda}$, the main difficulty is that we must consistently determine the traders' worst-off types who expect to be neither a buyer

\footnotetext{
${ }^{8}$ Notice that since ties occcur with zero probability, they will not affect expected quantities and so will be ignored in what follows.
} 
nor a seller as well as the allocation rules and, at the same time, maximize the objective function. Since ex ante any trader can be either a buyer or seller, optimally the worst-off types of traders are unlikely to have an extreme valuation and there can be a positive measure of such traders.

To simplify matters, we will assume that the distribution $F(v)$ is regular in the sense that $\alpha(\cdot \mid 1)$ and $\beta(\cdot \mid 1)$ are both strictly increasing ${ }^{9}$ and concentrate our attention on the more interesting type of bunching that is specific to ex ante unidentified traders. For a given $\lambda$, let $\bar{x}$ be such that $\beta(\bar{x} \mid \lambda)=\alpha(\bar{v} \mid \lambda)=\bar{v}$, and for any $x_{i} \in[\underline{v}, \bar{x}]$, let $y_{i}$ be such that $\alpha\left(y_{i} \mid \lambda\right)=\beta\left(x_{i} \mid \lambda\right)$. Define

$$
\delta\left(v_{i} \mid x_{i}, \lambda\right)= \begin{cases}\eta\left(v_{i} \mid x_{i}, \lambda\right), & \text { if } v_{i} \notin\left[x_{i}, y_{i}\right] \\ \beta\left(x_{i} \mid \lambda\right), & \text { if } v_{i} \in\left[x_{i}, y_{i}\right]\end{cases}
$$

then from the regularity assumption, $\delta\left(v_{i} \mid x_{i}, \lambda\right)$ is non-decreasing in $v_{i}$. We can now state and prove the main results of this paper.

Theorem 1. (a) Suppose that there exists an $x^{*}=\left(x_{1}^{*}, \cdots, x_{n}^{*}\right) \in[\underline{v}, \bar{x}]^{n}$ for which there exists at least one allocation $q=\left(q_{1}, \cdots, q_{n}\right)$ which satisfies

$$
\begin{aligned}
& q \text { solves }\left\{\begin{array}{l}
\max E\left[\sum_{i=1}^{n} \delta\left(v_{i} \mid x_{i}^{*}, \lambda\right) q_{i}(v)\right] \\
\text { s.t. }-k_{i} \leq q_{i} \leq k_{0}-k_{i} \text { for all } i \text { and } \sum_{i=1}^{n} q_{i}=0
\end{array}\right. \\
& \forall i, Q_{i}\left(v_{i}\right)=E_{-i}\left[q_{i}\left(v_{i}, v_{-i}\right)\right]=0 \text { for } v_{i} \in\left(x_{i}^{*}, y_{i}^{*}\right),
\end{aligned}
$$

then an allocation $q^{*}=\left(q_{1}^{*}, \cdots, q_{n}^{*}\right)$ is a solution to $\mathcal{P}_{\lambda}$ if and only if it satisfies $(A)$ and $(B)$ for this vector $x^{*}$. (b) If such a vector $x^{*}$ exists, it is unique.

Proof: (a) Sufficiency: Assume that $q^{*}$ satisfy (A) and (B) for some vector $x^{*} \in[\underline{v}, \bar{x}]^{n}$, then $q^{*}$ must be a solution to $\mathcal{P}_{\lambda}$.

The allocation $q^{*}$ solves (A) if and only if for any $v, q^{*}(v)$ satisfies:

$$
q_{i}^{*}\left(v_{i}, v_{-i}\right)= \begin{cases}k_{0}-k_{i}, & \text { if } \delta\left(v_{i} \mid x_{i}^{*}, \lambda\right)>l\left(v \mid x^{*}, \lambda\right) \\ \text { randomizing, } & \text { if } \delta\left(v_{i} \mid x_{i}^{*}, \lambda\right)=l\left(v \mid x^{*}, \lambda\right) \\ -k_{i}, & \text { otherwise }\end{cases}
$$

where $l\left(v \mid x^{*}, \lambda\right)$ for any $v$ is such that the number, $N_{1}$, of traders with types $v_{i}$ for which $\delta\left(v_{i} \mid x_{i}^{*}, \lambda\right) \geq$ $l\left(v \mid x^{*}, \lambda\right)$ is at least $n_{0}+1$, and the number, $N_{2}$, for which $\delta\left(v_{i} \mid x_{i}^{*}, \lambda\right)>l\left(v \mid x^{*}, \lambda\right)$ is at most $n_{0} \cdot{ }^{10}$

\footnotetext{
${ }^{9}$ This is the case for most common distributions. See, e.g., Myerson [14], for more details in the case where the distribution of valuations is not regular.

${ }^{10}$ The allocation $q^{*}$ as defined in (9) with any randomization rule solves the program in (A), but in order to satisfy (B), the randomization rule is strictly restricted and must be constructed in some specific way (see Lemma 4). Obviously, any allocation $q(v)$ differing from $q^{*}(v)$ only on a set of zero measure also solves the program in (A), but such differences will be ignored.
} 
Since $\delta\left(v_{i} \mid x_{i}^{*}, \lambda\right)$ is non-decreasing in $v_{i}$, then $q_{i}^{*}\left(v_{i}, v_{-i}\right)$ is non-decreasing in $v_{i}$, and so for $Q_{i}^{*}\left(v_{i}\right)$. Also, condition (B) implies that $x_{i}^{*} \in V^{*}\left(Q_{i}^{*}\right)$ for all $i$. Thus, $q^{*}$ satisfies all the constraints in $\mathcal{P}_{\lambda}$.

Now consider any alternative implementable allocation $\hat{q}=\left(\hat{q}_{1}, \cdots, \hat{q}_{n}\right)$, we have:

$$
\begin{aligned}
E\left[\sum_{i=1}^{n} \eta\left(v_{i} \mid v_{i}^{*}, \lambda\right) Q_{i}^{*}\left(v_{i}\right)\right] & =E\left[\sum_{i=1}^{n} \delta\left(v_{i} \mid x_{i}^{*}, \lambda\right) Q_{i}^{*}\left(v_{i}\right)\right] \\
& \geq E\left[\sum_{i=1}^{n} \delta\left(v_{i} \mid x_{i}^{*}, \lambda\right) \widehat{Q}_{i}\left(v_{i}\right)\right] \\
& \geq E\left[\sum_{i=1}^{n} \eta\left(v_{i} \mid \hat{v}_{i}, \lambda\right) \widehat{Q}_{i}\left(v_{i}\right)\right] .
\end{aligned}
$$

Equality (10) follows immediately from the fact that by definition, $\delta\left(v_{i} \mid x_{i}^{*}, \lambda\right)=\eta\left(v_{i} \mid v_{i}^{*}, \lambda\right)$ for $v_{i} \notin$ $\left[x_{i}^{*}, y_{i}^{*}\right]$ and from $(\mathrm{B}), Q_{i}^{*}\left(v_{i}\right)=0$ for $v_{i} \in\left(x_{i}^{*}, y_{i}^{*}\right)$. Inequality (11) follows immediately from the fact that $q^{*}$ satisfy (A). Inequality (12) follows from the fact that when $v_{i}<\hat{v}_{i}, \delta\left(v_{i} \mid x_{i}^{*}, \lambda\right) \leq \eta\left(v_{i} \mid \hat{v}_{i}, \lambda\right)$ and $\widehat{Q}_{i}\left(v_{i}\right) \leq 0$, and when $v_{i}>\hat{v}_{i}, \delta\left(v_{i} \mid x_{i}^{*}, \lambda\right) \geq \eta\left(v_{i} \mid \hat{v}_{i}, \lambda\right)$ and $\widehat{Q}_{i}\left(v_{i}\right) \geq 0$. Hence, $\delta\left(v_{i} \mid x_{i}^{*}, \lambda\right) \widehat{Q}_{i}\left(v_{i}\right) \geq$ $\eta\left(v_{i} \mid \hat{v}_{i}, \lambda\right) \widehat{Q}_{i}\left(v_{i}\right)$ for all $v_{i}$. Hence $q^{*}$ solves $\mathcal{P}_{\lambda}$.

Necessity: Now let $x^{*}$ and $q^{*}$ satisfy (A) and (B) and assume that some alternative solution $\hat{q}$ to $\mathcal{P}_{\lambda}$ exists. Clearly, (11) cannot hold with strict inequality: contradicting the assumption that $\hat{q}$ solves $\mathcal{P}_{\lambda}$. So $\hat{q}$ must solve the program in (A). Now suppose that $\hat{q}$ does not satisfy (B), i.e., for at least one $i$ there is a type $u \in\left(x_{i}^{*}, y_{i}^{*}\right)$ such that $\widehat{Q}_{i}(u) \neq 0$. Since $\widehat{Q}_{i}\left(v_{i}\right)$ is non-decreasing in $v_{i}$, then $\widehat{Q}_{i}\left(v_{i}\right)>0$ for all $v_{i} \in\left[u, y_{i}^{*}\right]$ or $\widehat{Q}_{i}\left(v_{i}\right)<0$ for all $v_{i} \in\left[x_{i}^{*}, u\right]$. If $\widehat{Q}_{i}(u)>0$, then $u \geq \max V^{*}\left(\widehat{Q}_{i}\right)$ and $\delta\left(v_{i} \mid x_{i}^{*}, \lambda\right)>\eta\left(v_{i} \mid \hat{v}_{i}, \lambda\right)$ for all $v_{i} \in\left(u, y_{i}^{*}\right)$ and $\hat{v}_{i} \in V^{*}\left(\widehat{Q}_{i}\right)$. If $\widehat{Q}_{i}(u)<0$, then $u \leq \min V^{*}\left(\widehat{Q}_{i}\right)$ and $\delta\left(v_{i} \mid x_{i}^{*}, \lambda\right)<\eta\left(v_{i} \mid \hat{v}_{i}, \lambda\right)$ for all $v_{i} \in\left(x_{i}^{*}, u\right)$ and $\hat{v}_{i} \in V^{*}\left(\widehat{Q}_{i}\right)$. Hence in both cases, the inequality in (12) is strict, which contradicts the assumption that $\hat{q}$ is a solution to $\mathcal{P}_{\lambda}$. Therefore, $\hat{q}$ must satisfy (A) and (B).

(b) Uniqueness: Suppose that $x^{*}$ and $z^{*}$ are two vectors satisfying the conditions in (a) and $x_{i}^{*} \neq z_{i}^{*}$ for at least one $i$. From (a), there exists an allocation $q^{*}$ satisfying (A) and (B) for $x^{*}$, so $q^{*}$ is a solution to $\mathcal{P}_{\lambda}$. Since $q^{*}$ solves $\mathcal{P}_{\lambda}, q^{*}$ must also satisfy (A) and (B) for $z^{*}$, but it is impossible because $x_{i}^{*} \neq z_{i}^{*}$.

Q.E.D

Theorem 1 characterizes the functional form of the solution to $\mathcal{P}_{\lambda}$. The optimal allocation can be expressed in terms of non-decreasing virtual valuations $\delta\left(v_{i} \mid x_{i}^{*}, \lambda\right)$ modified from $\eta\left(v_{i} \mid x_{i}^{*}, \lambda\right)$ for some appropriate values $\left(x_{1}^{*}, \cdots, x_{n}^{*}\right)$. Roughly speaking, the goods will be assigned to the traders whose 
modified virtual valuations are highest. The optimal allocation is designed in such a way that a high (low) type expects on average to be a net buyer (seller), and a positive mass of types in the middle expect to be neither buyer nor seller. Thus the bunching phenomena in intermediate ranges, which is here specific to ex ante unidentified traders, will be a general feature in the optimal mechanism even if the distribution of valuations is regular. Also from (9), when two traders or more have the same ranges of bunching in the optimal allocation, ties occur with positive probability and must be broken by randomizing. Unlike standard models where bunching is due to the irregularity of the distribution of valuations and ties can be broken by any randomization rule, now the tie breaking rule is strictly restricted: it must make types in the middle to have expected net trade zero. Hence the randomization rule becomes part and parcel of the design of the optimal allocation.

To complete the characterization of the solution to $\mathcal{P}_{\lambda}$, we next show how to compute the vector of worst-off types and the randomization rule by which ties can be broken.

Theorem 2. There exists a unique $x^{*}=\left(x_{1}^{*}, \cdots, x_{n}^{*}\right) \in[\underline{v}, \bar{x}]^{n}$ for which there will exist at least one randomization rule such that the allocation $q^{*}=\left(q_{1}^{*}, \cdots, q_{n}^{*}\right)$ as defined in (9) satisfies for all $i$, $Q_{i}^{*}\left(v_{i}\right)=E_{-i}\left[q_{i}^{*}\left(v_{i}, v_{-i}\right)\right]=0$ for $v_{i} \in\left[x_{i}^{*}, y_{i}^{*}\right]$.

In order to provide the basic intuition for Theorem 2, we consider the problem for 2 traders. For simplicity, we let $k_{0}=k_{1}+k_{2}$, where $k_{i}$ is the initial endowment of trader $i$. Here one trader receives all the units of the other. Without loss of generality, we let $k_{1} \leq k_{2}$. We wish to identify a set of $x_{i}^{*}$ 's and, if necessary, arandomization rule that satisfy the conditions of Theorem 2. One of two cases are possible, either traders share the same $x^{*}$ or they do not. We first suppose that they do and have the same modified virtual valuation function. Since traders share a common bunching range over which their virtual valuations are the same, there will be a positive probability of ties. Let $p$ be the probability that in such a case, the units are allocated to trader 1 . Since $Q_{i}^{*}\left(v_{i}\right)=E_{-i}\left[q_{i}^{*}\left(v_{i}, v_{-i}\right)\right]=0$ for $v_{i} \in\left[x^{*}, y^{*}\right]$ and for $i \in\{1,2\}$ (recall that $y^{*}$ is defined by $\alpha\left(y^{*} \mid \lambda\right)=\beta\left(x^{*} \mid \lambda\right)$ ), $p$ and $x^{*}$ must be such that the following holds:

$$
\begin{gathered}
k_{0} F\left(x^{*}\right)+k_{0} p\left[F\left(y^{*}\right)-F\left(x^{*}\right)\right]-k_{1}=0 \\
k_{0} F\left(x^{*}\right)+k_{0}(1-p)\left[F\left(y^{*}\right)-F\left(x^{*}\right)\right]-k_{2}=0
\end{gathered}
$$


Adding these two equations yields the following condition:

$$
2 k_{0} F\left(x^{*}\right)+k_{0}\left[F\left(y^{*}\right)-F\left(x^{*}\right)\right]=k_{1}+k_{2}=k_{0} \quad \text { or } \quad F\left(x^{*}\right)+F\left(y^{*}\right)=1
$$

Since $y^{*}$ is continuously increasing in $x^{*}$, there will exists a unique $x^{*}$ (and $y^{*}$ ) that satisfies the above condition. Note that $x^{*}$ is independent of $p$. So, the idea is to first find $x^{*}$ and $y^{*}$ using condition (15) and then calculate the probability $p$ so as to solve equation (13). If $k_{1}=k_{2}$, we need simply to let $p=1 / 2$. If $k_{1}<k_{0} / 2<k_{2}$, we need to move away from the symmetric randomization rule. As long as $\left[k_{1}-k_{0} F\left(x^{*}\right)\right]$ is non-negative, the conditions of Theorem 2 are met with $p=\frac{k_{1}-k_{0} F\left(x^{*}\right)}{k_{0}\left[F\left(y^{*}\right)-F\left(x^{*}\right)\right]}$. Here the randomization rule matters, any alternative rule will move us away from the zero-expected net trade condition.

However when $\left[k_{1}-k_{0} F\left(x^{*}\right)\right]$ is negative (which implies that $\left[k_{2}-k_{0} F\left(y^{*}\right)\right]$ is positive), there is no positive $p$ which satisfies equation (13) for $x^{*}$ and $y^{*}$. This will occur when the inital endowments are sufficiently asymmetric. In this case, we must have $x_{1}^{*}<x^{*}<x_{2}^{*}$ and the traders will not have the same virtual valuation function. Indeed, for all $v \in\left[x_{1}^{*}, y_{1}^{*}\right]$, we have $\delta\left(v \mid x_{1}^{*}, \lambda\right)<\delta\left(v \mid x_{2}^{*}, \lambda\right)$, so whenever $v_{1} \in\left[x_{1}^{*}, y_{1}^{*}\right]$, trader 1 of type $v_{1}$ wins only if $v_{2}<x_{1}^{*}$. Hence, we need to find $x_{1}^{*}$ such that $k_{1}=k_{0} F\left(x_{1}^{*}\right)$. Similarly, $y_{2}^{*}$ is such that $k_{2}=k_{0} F\left(y_{2}^{*}\right)$. Note that if $\left[k_{1}-k_{0} F\left(x^{*}\right)\right]$ is negative and $\left[k_{2}-k_{0} F\left(y^{*}\right)\right]$ is positive, we must have $x_{1}^{*}<x^{*}$ and $y_{2}^{*}>y^{*}$, which in turn implies that $x_{1}^{*}<x^{*}<x_{2}^{*}$. In this latter case, randomization rules do not matter since ties almost never occur, however the mechanism discriminates in favor of trader 2 since his virtual valuation function lies above that of trader 1 .

The formal proof of Theorem 2 is a generalization of the above example with an arbitrary number of traders. First, we find the vector of $x_{i}^{*}$ 's and in particular which groups of traders will share a same $x_{i}^{*}$ and modified virtual valuations. How to do this is explained in the Lemma 3, below. Second, for those groups of traders that share a same $x_{i}^{*}$ and have positive probability of ties, we must specify a randomization rule that induces zero-expected net trade. This is done in Lemma 4.

Proof: Without loss of generality, we can assume that $k_{1} \leq k_{2} \leq \cdots \leq k_{n}$. Since $\delta\left(x_{i}^{*} \mid x_{i}^{*}, \lambda\right)<$ $\delta\left(x_{j}^{*} \mid x_{j}^{*}, \lambda\right)$ when $x_{i}^{*}<x_{j}^{*}$, from $(9)$, we are clearly looking for a vector $x^{*}$ such that $x_{1}^{*} \leq x_{2}^{*} \leq \cdots \leq$ $x_{n}^{*}$.

For now, consider the solution to (A) $\tilde{q}(v)$ (given some $x^{*}$ with $x_{1}^{*} \leq x_{2}^{*} \leq \cdots \leq x_{n}^{*}$ ) where ties 
are always broken in favor of those with the highest indexes. Let $\widetilde{Q}_{i}\left(x_{i}^{*}\right)=E_{-i}\left[\tilde{q}_{i}\left(x_{i}^{*}, v_{-i}\right)\right]$ be the expected net trade for participant $i$ of type $x_{i}^{*}$ under this alloaction rule (generally, $\widetilde{Q}_{i}\left(x_{i}^{*}\right) \neq 0$ ). $\widetilde{Q}_{i}\left(x_{i}^{*}\right)$ is well-defined and independent of $x_{-i}^{*}$ provided the rank of $x_{i}^{*}$ is preserved. $^{11}$

Lemma 3. (a) A vector $x^{*}$ with $x_{1}^{*} \leq x_{2}^{*} \leq \cdots \leq x_{n}^{*}$ satisfies the conditions of Theorem 2, only if it is such that whenever $x_{l}^{*}=x_{S}^{*} \forall l \in S=\{i, \cdots, j\}$ with $i \leq j$ and $x_{l}^{*} \neq x_{S}^{*} \forall l \notin S$, we have $\sum_{l=i}^{m} \widetilde{Q}_{l}\left(x_{S}^{*}\right) \leq 0$ for all $i \leq m<j$ and $\sum_{l=i}^{j} \widetilde{Q}_{l}\left(x_{S}^{*}\right)=0$. (b) Such a vector $x^{*}$ exists.

Proof of Lemma 3: (a) Suppose that $x^{*}$ is a vector for which there exist at least one randomization rule such that the allocation $q^{*}=\left(q_{1}^{*}, \cdots, q_{n}^{*}\right)$ as defined in $(9)$ satisfies for all $i, Q_{i}^{*}\left(v_{i}\right)=0$ for $v_{i} \in\left[x_{i}^{*}, y_{i}^{*}\right]$. Note that the $\delta\left(v_{i} \mid x_{i}^{*}, \lambda\right)$ increases up to $x_{i}^{*}$, then is constant between $x_{i}^{*}$ and $y_{i}^{*}$, and increases afterward. Thus, there is a positive probability of ties between $i$ and $j$ if and only if $x_{i}^{*}=x_{j}^{*}$.

When $x_{j}^{*} \neq x_{i}^{*} \forall j \neq i$, since the probability of ties between $i$ of type $x_{i}^{*}$ and any other participants is zero, we must have $\widetilde{Q}_{i}\left(x_{i}^{*}\right)=Q_{i}^{*}\left(x_{i}^{*}\right)=0$. Now consider a subset of participants, $S=\{i, \cdots, j\}$ with $i<j$, we have $x_{l}^{*}=x_{S}^{*} \forall l \in S$ and $x_{l}^{*} \neq x_{S}^{*} \forall l \notin S$. Participants in $S$ will have a strict positive probability of ties with and only with other members of $S$. In such a case, we have $\widetilde{Q}_{i}\left(x_{S}^{*}\right) \leq Q_{i}^{*}\left(x_{S}^{*}\right)=$ 0 because $i$ of type $x_{S}^{*}$ always loses ties against participants of higher indexes in $S$ in the solution leading to $\widetilde{Q}_{i}\left(x_{S}^{*}\right)$. Similarly, since all $l \in\{i, \cdots, m\}(i \leq m \leq j)$ always loses ties against participants of indexes higher than $m$ in the solution leading to $\widetilde{Q}_{l}\left(x_{S}^{*}\right)$, and the total expected net trade for the group of participants $\{i, \cdots, m\}$ of type $x_{S}^{*}$ is independent of how ties are randomly broken between them, we have $\sum_{l=i}^{m} \widetilde{Q}_{l}\left(x_{S}^{*}\right) \leq \sum_{l=i}^{m} Q_{l}^{*}\left(x_{S}^{*}\right)=0$. Finally, for $m=j$, since the probability of ties between any $l \in S$ and any $h \notin S$ is zero, we must have $\sum_{l=i}^{j} \widetilde{Q}_{l}\left(x_{S}^{*}\right)=\sum_{l=i}^{j} Q_{l}^{*}\left(x_{S}^{*}\right)=0$.

(b) We show by construction the existence of a unique $x^{*}$ which satsifies the conditions of Lemma

\footnotetext{
${ }^{11}$ Formally, we define for all $x_{i} \in[\underline{v}, \bar{v}]$

$$
\begin{aligned}
\widetilde{Q}_{i}\left(x_{i}\right)= & k_{0} \operatorname{Pr}\left\{\text { there are less than } n_{0} \text { other traders who have either valuation greater than } y_{i}\right. \text { and } \\
& \text { index lower than } \left.i \text { or valuation greater than } x_{i} \text { and index higher than } i\right\} \\
& +r \operatorname{Pr}\left\{\text { there are exactly } n_{0} \text { other traders who have either valuation greater than } y_{i}\right. \text { and } \\
& \text { index lower than } \left.i \text { or valuation greater than } x_{i} \text { and index higher than } i\right\} \\
& -k_{i}
\end{aligned}
$$
}

where $y_{i}$ is such that $\alpha\left(y_{i} \mid \lambda\right)=\beta\left(x_{i} \mid \lambda\right)$ if $x_{i} \leq \bar{x}$ and $y_{i}=\bar{v}$ if $x_{i}>\bar{x}$. Then for any $x^{*}$ with $x_{1}^{*} \leq \cdots \leq x_{n}^{*}, \widetilde{Q}_{i}\left(x_{i}^{*}\right)$ is the expected net trade under allocation $\tilde{q}$. 
3. We first find recursively the vector $\tilde{x}=\left(\tilde{x}_{1}, \cdots, \tilde{x}_{n}\right)$ such that for all $l,{ }^{12}$

$$
\sum_{i=1}^{l} \widetilde{Q}_{i}\left(\min \left(\tilde{x}_{i}, \cdots, \tilde{x}_{l}\right)\right)=0 \text { or } \tilde{x}_{l}=\bar{x} \text { if } \sum_{i=1}^{l} \widetilde{Q}_{i}\left(\min \left(\tilde{x}_{i}, \cdots, \tilde{x}_{l}\right)\right)<0 \forall \tilde{x}_{l}<\bar{x}
$$

First note that traders with index $i \leq l$ and with types between $\tilde{x}_{i}=\underline{v}$ and the corresponding $y_{i}$, will always have virtual valuations less than those of the other traders, they will collectively always be a source of units and their expected net trade will be negative: $\sum_{i=1}^{l} \widetilde{Q}_{i}(\underline{v}) \leq 0$.

Since $\widetilde{Q}_{1}(\underline{v}) \leq 0$, strict monotonicity and continuity of $\widetilde{Q}_{1}(\cdot)$ imply the existence of a unique solution $\tilde{x}_{1}$. Given this $\tilde{x}_{1}$, we next find the unique $\tilde{x}_{2}$ such that $\widetilde{Q}_{1}\left(\min \left(\tilde{x}_{1}, \tilde{x}_{2}\right)\right)+\widetilde{Q}_{2}\left(\tilde{x}_{2}\right)=0$, or $\tilde{x}_{2}=\bar{x}$ if it is negative for all $\tilde{x}_{2}<\bar{x}$. Again, $\widetilde{Q}_{1}(\underline{v})+\widetilde{Q}_{2}(\underline{v}) \leq 0$, so there exists a unique solution $\tilde{x}_{2}$. We then proceed recursively to find all $\tilde{x}_{l}, l \leq n-1$. Finally, given $\tilde{x}_{1}, \cdots, \tilde{x}_{n-1}$ we solve for $\tilde{x}_{n}$. Note that when $\tilde{x}_{n}=\underline{v}$, we have $\sum_{i=1}^{n} \widetilde{Q}_{i}\left(\min \left(\tilde{x}_{i}, \cdots, \tilde{x}_{n}\right)\right)=\sum_{i=1}^{n} \widetilde{Q}_{i}(\underline{v}) \leq 0$. Now suppose that $\tilde{x}_{n}=\bar{x}$ and let $l<n$ be the highest index such that $\tilde{x}_{l}<\bar{x}$. We have $\sum_{i=1}^{n} \widetilde{Q}_{i}\left(\min \left(\tilde{x}_{i}, \cdots, \tilde{x}_{n}\right)\right)=$ $\sum_{i=1}^{l} \widetilde{Q}_{i}\left(\min \left(\tilde{x}_{i}, \cdots, \tilde{x}_{l}\right)\right)+\sum_{i=l+1}^{n} \widetilde{Q}_{i}(\bar{x})=\sum_{i=1}^{n} \widetilde{Q}_{i}(\bar{x}) \geq 0$. The last inequality follows because traders with the highest indexes and with types between $\bar{x}$ and $\bar{v}$, will always have virtual valuations higher than those of the other traders, they will collectively always be a sink of units with positive expected net trade. Hence, there exists a unique $\tilde{x}_{n} \in[\underline{v}, \bar{x}]$ which solves the $n^{\text {th }}$ equation in (14).

Next we set $x_{i}^{*}=\min \left(\tilde{x}_{i}, \cdots, \tilde{x}_{n}\right)$. One can verify that by construction $x_{1}^{*} \leq \cdots \leq x_{n}^{*} \leq \tilde{x}_{n} \leq \bar{x}$. Now suppose that $x_{l}^{*}=x_{S}^{*} \forall i \leq l \leq j, x_{l}^{*}<x_{S}^{*} \forall l \leq i-1$, and $x_{l}^{*}>x_{S}^{*} \forall l \geq j+1$. From this assumption, $x_{l}^{*}=\min \left(\tilde{x}_{l}, \cdots, \tilde{x}_{i-1}\right)$ for $1 \leq l \leq i-1$ and $x_{l}^{*}=\min \left(\tilde{x}_{l}, \cdots, \tilde{x}_{j}\right) \leq \min \left(\tilde{x}_{l}, \cdots, \tilde{x}_{m}\right)$ for $1 \leq l \leq m \leq j$. In particular, we have $\tilde{x}_{i-1}=x_{i-1}^{*}<\bar{x}$ and $\tilde{x}_{j}=x_{j}^{*}<\bar{x}$, which imply that $\sum_{l=1}^{i-1} \widetilde{Q}_{l}\left(\min \left(\tilde{x}_{l}, \cdots, \tilde{x}_{i-1}\right)\right)=0$ and $\sum_{l=1}^{j} \widetilde{Q}_{l}\left(\min \left(\tilde{x}_{l}, \cdots, \tilde{x}_{j}\right)\right)=0$. Hence

$$
\begin{aligned}
\sum_{l=i}^{m} \widetilde{Q}_{l}\left(x_{l}^{*}\right) & =\sum_{l=1}^{m} \widetilde{Q}_{l}\left(x_{l}^{*}\right)-\sum_{l=1}^{i-1} \widetilde{Q}_{l}\left(x_{l}^{*}\right) \\
& \leq \sum_{l=1}^{m} \widetilde{Q}_{l}\left(\min \left(\tilde{x}_{l}, \cdots, \tilde{x}_{m}\right)\right)-\sum_{l=1}^{i-1} \widetilde{Q}_{l}\left(\min \left(\tilde{x}_{l}, \cdots, \tilde{x}_{i-1}\right)\right) \\
& \leq 0
\end{aligned}
$$

where the last two inequalities hold with equalities when $m=j$.

Q.E.D

\footnotetext{
${ }^{12}$ Each $\widetilde{Q}_{i}\left(\tilde{x}_{i}\right)$ is as defined in footnote 11 . It is easy to verify that $\widetilde{Q}_{i}\left(\tilde{x}_{i}\right)$ is continous and strictly increasing on $[\underline{v}, \bar{v}]$ for all $i \leq n-1$, and $\widetilde{Q}_{n}\left(\tilde{x}_{n}\right)$ is continous and strictly increasing on $[\underline{v}, \bar{x}]$ and constant on $[\bar{x}, \bar{v}]$.
} 
To complete the proof of Theorem 2, it remains to prove that given the vector $x^{*}$ satisfying the condition of Lemma 3, a randomization rule can be find. The formal proof of the following lemma is given in the appendix.

Lemma 4. If a vector $x^{*}$ satisfy the conditions of Lemma 3, then there exists a randomization rule such that $q^{*}$ as defined in (9) satisfies for all $i, Q_{i}^{*}\left(v_{i}\right)=0$ for $v_{i} \in\left[x_{i}^{*}, y_{i}^{*}\right]$.

Theorems 1 and 2 prove a complete characterization of the optimal allocation. In the terminology of Wilson [22], the optimal allocation can be called as a generalized double auction in virtual valuations that maximizes the expected gains from trade measured in the virtual valuations $\delta\left(v_{i} \mid x_{i}^{*}, \lambda\right)$ for the traders. What is particular here is that the tie-breaking rule now affects the traders' expected net trades, hence it becomes an important instrument in the design of the optimal trading mechanism in order to make the types in the middle to have expected net trade zero.

In the following theorem, we provide two basic comparative results.

Theorem 3. The weighted sum $W_{\lambda}\left(q^{*}\right)$ of the expected gains from trade and the expected revenue from the optimal allocation is (i) strictly increasing with $k_{0}$ and (ii) non-decreasing as the initial endowments become more symmetric.

Proof: (i) To show that $W_{\lambda}\left(q^{*}\right)$ is strictly increasing in $k_{0}$, notice that the optimal allocation $q^{*}$ given some initial $k_{0}^{\prime}$ is feasible with a higher $k_{0}^{\prime \prime}>k_{0}^{\prime}$. Furthermore, $q^{*}$ is not optimal since $q^{*}$ cannot satisfy condition (A) of Theorem 1 with $k_{0}^{\prime \prime}$. (In the allocation $q^{*}$, only $k_{0}^{\prime}-k_{i}$ units are transfered to trader $i$ when his valuation is high, but optimally the net number of units bought by the trader should be $k_{0}^{\prime \prime}-k_{i}$.) There must exist a $\hat{q}$ which generates higher value for $W_{\lambda}$.

(ii) Let $q^{*}$ be the optimal allocation with initial endowments $\left(k_{1}, \cdots, k_{n}\right)$. Now suppose that we reallocate the initial endowment so that $k_{i}^{a}=a k_{i}+(1-a) k_{j}, k_{j}^{a}=(1-a) k_{i}+a k_{j}$ for some $a \in(0,1)$, and $k_{l}^{a}=k_{l}$ for $l \neq i, j$. First, we define an allocation $q^{a}$ as follows. For any $v$, let $w$ be the vector with $w_{i}=v_{j}, w_{j}=v_{i}$, and $w_{l}=v_{l}$ for $l \neq i, j$ and

$$
\begin{aligned}
& q_{i}^{a}(v)=a q_{i}^{*}(v)+(1-a) q_{j}^{*}(w) \\
& q_{j}^{a}(v)=a q_{j}^{*}(v)+(1-a) q_{i}^{*}(w) \\
& q_{l}^{a}(v)=a q_{l}^{*}(v)+(1-a) q_{l}^{*}(w) \quad \forall l \neq i, j,
\end{aligned}
$$

then $q^{a}$ is implementable. Indeed, $\sum_{l=1}^{n} q_{l}^{a}(v)=a \sum_{l=1}^{n} q_{l}^{*}(v)+(1-a) \sum_{l=1}^{n} q_{l}^{*}(w)=0 ;-k_{l} \leq q_{l}^{*} \leq$ $k_{0}-k_{l}$ for all $l$ imply $-k_{l}^{a} \leq q_{l}^{a} \leq k_{0}-k_{l}^{a}$ for all $l$; and finally since all traders have a common 
distribution of valuations, we have $Q_{i}^{a}\left(v_{i}\right)=a Q_{i}^{*}\left(v_{i}\right)+(1-a) Q_{j}^{*}\left(v_{i}\right), Q_{j}^{a}\left(v_{j}\right)=a Q_{j}^{*}\left(v_{j}\right)+(1-a) Q_{i}^{*}\left(v_{j}\right)$, and $Q_{l}^{a}\left(v_{l}\right)=Q_{l}^{*}\left(v_{l}\right)$ for $l \neq i, j$. Hence $Q_{l}^{a}\left(v_{l}\right)$ is also non-decreasing for all $l$ and there must be an incentive feasible mechanism implementing $q^{a}$.

Second, we have for $v_{l}^{*} \in V^{*}\left(Q_{l}^{*}\right)$ and $v_{l}^{a} \in V^{*}\left(Q_{l}^{a}\right)$,

$$
\begin{aligned}
W_{\lambda}\left(q^{a}\right)-W_{\lambda}\left(q^{*}\right) \\
=\sum_{l=1}^{n} E_{l}\left[\eta\left(v_{l} \mid v_{l}^{a}, \lambda\right) Q_{l}^{a}\left(v_{l}\right)\right]-\sum_{l=1}^{n} E_{l}\left[\eta\left(v_{l} \mid v_{l}^{*}, \lambda\right) Q_{l}^{*}\left(v_{l}\right)\right] \\
=\quad E_{i}\left[\eta\left(v_{i} \mid v_{i}^{a}, \lambda\right) Q_{i}^{a}\left(v_{i}\right)\right]+E_{j}\left[\eta\left(v_{j} \mid v_{j}^{a}, \lambda\right) Q_{j}^{a}\left(v_{j}\right)\right]-E_{i}\left[\eta\left(v_{i} \mid v_{i}^{*}, \lambda\right) Q_{i}^{*}\left(v_{i}\right)\right]-E_{j}\left[\eta\left(v_{j} \mid v_{j}^{*}, \lambda\right) Q_{j}^{*}\left(v_{j}\right)\right] \\
=E_{i}\left[\eta\left(v_{i} \mid v_{i}^{a}, \lambda\right)\left(a Q_{i}^{*}\left(v_{i}\right)+(1-a) Q_{j}^{*}\left(v_{i}\right)\right)\right]+E_{j}\left[\eta\left(v_{j} \mid v_{j}^{a}, \lambda\right)\left(a Q_{j}^{*}\left(v_{j}\right)+(1-a) Q_{i}^{*}\left(v_{j}\right)\right)\right] \\
\quad-E_{i}\left[\eta\left(v_{i} \mid v_{i}^{*}, \lambda\right) Q_{i}^{*}\left(v_{i}\right)\right]-E_{j}\left[\eta\left(v_{j} \mid v_{j}^{*}, \lambda\right) Q_{j}^{*}\left(v_{j}\right)\right] \\
=\quad E_{i}\left[\left(a \eta\left(v_{i} \mid v_{i}^{a}, \lambda\right)+(1-a) \eta\left(v_{i} \mid v_{j}^{a}, \lambda\right)-\eta\left(v_{i} \mid v_{i}^{*}, \lambda\right)\right) Q_{i}^{*}\left(v_{i}\right)\right] \\
\quad+E_{j}\left[\left((1-a) \eta\left(v_{j} \mid v_{i}^{a}, \lambda\right)+a \eta\left(v_{j} \mid v_{j}^{a}, \lambda\right)-\eta\left(v_{j} \mid v_{j}^{*}, \lambda\right)\right) Q_{j}^{*}\left(v_{j}\right)\right] \\
\geq \quad 0
\end{aligned}
$$

The last inequality follows from the fact that for any given $\hat{v},\left(\eta\left(v_{l} \mid \hat{v}, \lambda\right)-\eta\left(v_{l} \mid v_{l}^{*}, \lambda\right)\right) Q_{l}^{*}\left(v_{l}\right) \geq 0$ for $l=i, j$. Therefore, $W_{\lambda}\left(q^{a}\right) \geq W_{\lambda}\left(q^{*}\right) .{ }^{13}$ This completes our proof.

Q.E.D

Intuitively, since the optimal allocation requires that all goods go to the traders whose virtual valuations are highest, then when the traders have a higher level of demand, the goods will be assigned to a smaller number of traders with the highest virtual valuations and $W_{\lambda}\left(q^{*}\right)$, which is the expected gains from trade from the optimal allocation measured in virtual valuations, increases. On the other hand, each trader may bid either higher or lower than his true valuation, depending on whether he is more likely to sell or buy in the ultimate transaction; the cost of satisfying informational incentive constraints depends on the trader's types partition (low types to be sellers, high types to be buyers, and to be neither buyers nor sellers between them) and decreases as the partition becomes more symmetric. This implies that when the initial endowments are more evenly distributed amongst the traders, the expected information rents accruing to all traders can be reduced and there may be more expected revenue from the trading mechanism yielding a higher $W_{\lambda}$. This intuition also

\footnotetext{
${ }^{13}$ The inequality is strict if the ranges over which $Q_{i}^{*}\left(v_{i}\right)=0$ and the ranges over which $Q_{j}^{*}\left(v_{j}\right)=0$ do not coincide. So, if initially $i$ and $j$ have different bunching intervals, $W_{\lambda}\left(q^{*}\right)$ strictly increases as the initial endowments become more symmetric between $i$ and $j$; but if $\left|k_{j}-k_{i}\right|$ is small so that their bunching ranges are the same, a more symmetric distribution of the endowments between $i$ and $j$ does not alter their common bunching interval and nor $W_{\lambda}$ because $q^{a}$ is the optimal allocation with $k_{i}^{a}$ and $k_{j}^{a}$.
} 
gives an explanation to the result of the next section that the expected gains from trade from the ex ante efficient allocation is non-decreasing as the initial endowments become more symmetric, as well as the result of Cramton et al. [2] that there is a link between ex post efficient mechanisms and symmetry of endowments.

\section{Ex Ante Efficient Mechanisms}

In this section, we show that the optimal allocation constructed in Theorems 1 and 2 can be useful to characterize the most efficient trading mechanism subject to the constraint that traders are not subsidized. Cramton et al. [2] examine a special case of this trading problem in which traders have the highest level of demand possible (the economy wide endowment). Despite incomplete information about the valuation of the object, they show that ex post efficiency can be achieved when the object is initially jointly owned, with no player having too large a share. Lu [8] extends the work of Cramton et al. by considering more standard preferences, and shows that the implementability of ex post efficient allocations is primarily determined by the degree of revelation of private information required in achieving it. In the case of linear demand, for example, the achievement of ex post efficiency requires full revelation of private information, and it is impossible to have an incentive feasible mechanism that is ex post efficient except when all traders are ex ante symmetric. When the traders have inelastic demands for a fixed number of units, partial revelation of private information is sufficient to allocate the goods efficiently and this allows for ex post efficiency to be possible as in Cramton et al. when the traders are sufficiently symmetric.

Since ex post efficiency may not be achieved by any incentive feasible mechanism in asymmetric economic environments, unless some outsider is willing to provide a subsidy to the traders for participating in the trading mechanism, it is natural to seek a mechanism that maximizes expected total gains from trade, subject to the incentive compatibility and individual rationality constraints, as well as the market-maker's budget constraint. That is, we are looking for the ex ante efficient 
mechanism that solves ${ }^{14}$

$$
\mathcal{P}_{s}\left\{\begin{array}{l}
\max E\left[\sum_{i=1}^{n} v_{i} q_{i}(v)\right] \\
\text { s.t. }-k_{i} \leq q_{i} \leq k_{0}-k_{i} \text { and } \sum_{i=1}^{n} q_{i}=0 \\
\{q, t\} \text { is incentive feasible and } \sum_{i=1}^{n} t_{i}=0
\end{array}\right.
$$

The following result that is an adaptation of Theorem 1 of Myerson and Satterthwaite [15] and Lemma 4 of Cramton et al. [2], allows to remove the payment function from problem $\mathcal{P}_{s}$. The proof is given in the Appendix.

Lemma 5. For any allocation $q$ such that $Q_{i}\left(v_{i}\right)$ is non-decreasing for all $i \in N$, there exists a payment function $t$ with $\sum_{i=1}^{n} t_{i}=0$ such that $\{q, t\}$ is incentive feasible if and only if $E\left[\sum_{i=1}^{n} \eta\left(v_{i} \mid v_{i}^{*}\right) q_{i}(v)\right] \geq$ 0 , where $v_{i}^{*} \in V^{*}\left(Q_{i}\right){ }^{15}$

From Lemmas 1 and 5, problem $\mathcal{P}_{s}$ can be rewritten as

$$
\mathcal{P}_{s}\left\{\begin{array}{l}
\max E\left[\sum_{i=1}^{n} v_{i} q_{i}(v)\right] \\
\text { s.t. }-k_{i} \leq q_{i} \leq k_{0}-k_{i} \text { and } \sum_{i=1}^{n} q_{i}=0 \\
Q_{i}\left(v_{i}\right) \text { is non-decreasing and } E\left[\sum_{i=1}^{n} \eta\left(v_{i} \mid v_{i}^{*}\right) q_{i}(v)\right] \geq 0, \text { where } v_{i}^{*} \in V^{*}\left(Q_{i}\right) .
\end{array}\right.
$$

The following theorem proves that all solution to $\mathcal{P}_{s}$ is a solution to $\mathcal{P}_{\lambda}$ for some $\lambda$.

Theorem 4. There exists a $\lambda \in[0,1)$ such that one allocation $q$ is a solution to $\mathcal{P}_{s}$ if and only if $q$ solves $\mathcal{P}_{\lambda}$.

Proof: If the ex post efficient allocation $q^{e}$ satisfies $E\left[\sum_{i=1}^{n} \eta\left(v_{i} \mid v_{i}^{*}\right) q_{i}^{e}(v)\right] \geq 0$ where $v_{i}^{*} \in V^{*}\left(Q_{i}^{e}\right)$, then $q^{e}$ is the solution to $\mathcal{P}_{s}$, but it also solves $\mathcal{P}_{\lambda}$ for $\lambda=0$. Now assume that $E\left[\sum_{i=1}^{n} \eta\left(v_{i} \mid v_{i}^{*}\right) q_{i}^{e}(v)\right]<$ 0 . Let $q^{*}(v \mid \lambda)$ be the solution to $\mathcal{P}_{\lambda}$ for some given $\lambda \in[0,1]$. We argue that there exists a $\lambda$ such that $q^{*}(v \mid \lambda)$ satisfies: ${ }^{16}$

$$
E\left[\sum_{i=1}^{n} \eta\left(v_{i} \mid x_{i}^{*}\right) q_{i}(v)\right]=0, \text { where } x_{i}^{*}=\min V^{*}\left(Q_{i}\right)
$$

The existence of $\lambda$ such that $q^{*}(v \mid \lambda)$ satisfies (15) follows from the fact that the expected revenue

\footnotetext{
${ }^{14}$ See, e.g., Holmström and Myerson [4]. Here we focus just on the ex ante efficient mechanism that places equal welfare weights on every trader and maximizes the sum of all traders' expected gains from trade. This maximization is equivalent to maximizing the sum of the traders' ex ante expected utilities because each trader's utility function is separable in money and his valuation.

${ }^{15}$ As a corollary of Lemma 5, there exists an incentive feasible mechanism that can implement the ex post efficient allocation $q^{e}$ as defined in (8) while satisfying budget constraint if and only if $\sum_{i=1}^{n} E\left[\eta\left(v_{i} \mid v_{i}^{*}\right) q_{i}^{e}(v)\right] \geq 0$, where $v_{i}^{*} \in V^{*}\left(Q_{i}^{e}\right)$. Lu [8] shows that given $k_{0}$-unit demand, the set of endowments for which ex post efficiency is possible is a convex symmetric set centered around the most evenly distributed endowment $\left(\frac{K}{n}, \cdots, \frac{K}{n}\right)$.

${ }^{16}$ As one would expect, there is only one such $\lambda$. The proof of this is ommitted.
} 
is continuous in $\lambda,{ }^{17}$ negative when $\lambda=0$, and positive when $\lambda=1$. Now recall that $q^{*}(v \mid \lambda)$ maximizes $W_{\lambda}(\cdot)$ a weighted sum of expected gains from trade and the market-maker's revenue. So any alternative implementable allocation $q$ would either generate less (i.e. negative) revenue to the market-maker and hence not satisfy the budget balance condition, or would generate less gains from trade. $q^{*}(v \mid \lambda)$ is indeed the solution to $\mathcal{P}_{s}$.

Q.E.D

From Theorem 4, maximizing expected gains from trade, subject to incentive feasibility and budget constraints, is equivalent to maximizing a specific weighted sum of expected gains from trade and the market-maker's revenue. The ex ante efficient allocation is also a generalized double auction in $\lambda$-virtual valuations for some $\lambda$ such that the market-maker's expected revenue equals zero. Since the solution $q^{*}(v \mid 1)$ to $\mathcal{P}_{1}$ always generates a positive revenue $E\left[\sum_{i=1}^{n} \delta\left(v_{i} \mid x_{i}^{*}\right) q_{i}^{*}(v \mid 1)\right]>0$, so the revenue-maximizing allocation can never be ex ante efficient. We can make a comparison between a market-maker in this exchange economy and a monopoly seller. Here the ex ante efficient allocation is socially optimal, but it is not optimal for the market-maker who will sacrifice some efficiency for the purpose of rent extraction. It is exactly what a profit-maximizing monopoly does in the usual context.

Given $k_{0}$-unit demand and initial endowments $\left(k_{1}, \cdots, k_{n}\right)$, let $G_{a}$ be the expected total gains from trade of ex ante efficient allocations (the maximum gain from trade given the incentive feasibility constraints and the balanced budget constraint).

Theorem 5. The expected total gains from trade from the ex ante efficient allocation $G_{a}$ is (i) strictly increasing with $k_{0}$ and (ii) non-decreasing as the initial endowments become more symmetric.

Proof: This proof is similar to the proof of Theorem 3 except that we must additionally check the budget balance condition.

(i) To show that $G_{a}$ is strictly increasing in $k_{0}$, notice that the solution $q^{*}$ to $\mathcal{P}_{s}$ given some initial $k_{0}^{\prime}$ is implementable with a higher $k_{0}^{\prime \prime}>k_{0}^{\prime}$. Furthermore, $q^{*}$ is not optimal since $q^{*}$ cannot be a solution to $\mathcal{P}_{\lambda}$ with $k_{0}^{\prime \prime}$ for any $\lambda$. There must exist a $\hat{q}$ which generates more gains from trade.

(ii) As in the proof of Theorem 3 , let $q^{*}$ be the solution to $\mathcal{P}_{s}$ with initial endowments $k_{i}$ and $k_{j}$,

\footnotetext{
${ }^{17}$ To see that $E\left[\sum_{i=1}^{n} \eta\left(v_{i} \mid x_{i}^{*}(\lambda)\right) Q_{i}^{*}(v \mid \lambda)\right]$ with $x_{i}^{*}(\lambda)=\min V^{*}\left(Q_{i}^{*}(\cdot \mid \lambda)\right)$ is continuous, observe that from $\alpha\left(y_{i}, \lambda\right)=$ $\beta\left(x_{i}, \lambda\right), y_{i}$ as a function of $x_{i}$ and $\lambda$ is continuous, which implies that the function $\widetilde{Q}_{i}\left(x_{i}, \lambda\right)$ is continuous in $x_{i}$ and $\lambda$. Thus, the solution $\tilde{x}$ to (14) is continuous in $\lambda$, and so for $x^{*}$. Further, the continuity of $x^{*}$ in $\lambda$ implies that $\delta\left(v_{i} \mid x_{i}^{*}\right)$ and $Q_{i}^{*}\left(v_{i} \mid \lambda\right)$ are continuous in $\lambda$. So $E\left[\sum_{i=1}^{n} \delta\left(v_{i} \mid x_{i}^{*}(\lambda)\right) Q_{i}^{*}\left(v_{i} \mid \lambda\right)\right]$ must be continuous in $\lambda$.
} 
and reallocate the initial endowment and define the allocation $q^{a}$. It is shown that $q^{a}$ is implementable with the reallocated endowments and generates at least as much revenue as $q^{*}$; thus $q^{a}$ satisfies the budget constraint $E\left[\sum_{l=1}^{n} \eta\left(v_{l} \mid v_{l}^{a}\right) q_{l}^{a}(v)\right] \geq 0$. Further, since $Q_{i}^{a}\left(v_{i}\right)=a Q_{i}^{*}\left(v_{i}\right)+(1-a) Q_{j}^{*}\left(v_{i}\right)$, $Q_{j}^{a}\left(v_{j}\right)=a Q_{j}^{*}\left(v_{j}\right)+(1-a) Q_{i}^{*}\left(v_{j}\right)$, and $Q_{l}^{a}\left(v_{l}\right)=Q_{l}^{*}\left(v_{l}\right)$ for $l \neq i, j$, and all traders have a common distribution of valuations, then

$$
E\left[\sum_{l=1}^{n} v_{l} Q_{l}^{a}\left(v_{l}\right)\right]=\sum_{l=1}^{n} E_{l}\left[v_{l} Q_{l}^{*}\left(v_{l}\right)\right] .
$$

Thus $q^{a}$ generates as much gains from trade as $q^{*}$, which implies that $G_{a}$ is non-decreasing as the initial endowments become more symmetric.

Q.E.D

\section{Convergence to Bid-ask Mechanisms}

We have characterized the optimal allocation in the context of an exchange economy when there are few participants and everyone takes advantage of opportunities to affect the terms of trade. We consider in this section, how the optimal allocation changes as the number of traders increases. More precisely, our aim is to establish that, provided the number of traders is large enough, the optimal trading mechanism converges toward a bid-ask mechanism.

Given an $n$-player trading problem $N$, let denote $n_{l}$ the number of traders each of whom has an endowment of $l$ units. Let $\tau N$ be the $\tau$-fold replication of $N$. That is, there are $\tau n$ traders, with $\tau n_{l}$ traders owning $l$ units of the traded good. The total number of units in $\tau N$ is $\tau K$. In order to evaluate the objective function $W_{\lambda}$ when $\tau \rightarrow \infty$, we divide it by the number of replication. For all $\tau=2,3, \cdots, \infty$ and $Q=\left(Q_{1}, \cdots, Q_{\tau n}\right), \operatorname{let}^{18}$

$$
\widetilde{W}_{\lambda}(Q)=\frac{1}{\tau} W_{\lambda}(Q)=\frac{1}{\tau} E\left[\sum_{i=1}^{n \tau} \eta\left(v_{i} \mid v_{i}^{*}, \lambda\right) Q_{i}\left(v_{i}\right)\right], \quad \text { where } v_{i}^{*} \in V^{*}\left(Q_{i}\right) .
$$

Note that if for all $v$, we have $Q_{i}(v)=Q_{j}(v)$ whenever $k_{i}=k_{j}$, then we can simply write $\widetilde{W}_{\lambda}(Q)=$ $E\left[\sum_{i \in N} \eta\left(v_{i} \mid v_{i}^{*}, \lambda\right) Q_{i}\left(v_{i}\right)\right]$.

For some given $\lambda$, let $\hat{x} \in[\underline{v}, \bar{x}]$ be defined by $\left(n k_{0}-K\right)(1-F(\hat{y}))=K F(\hat{x})$ with $\alpha(\hat{y} \mid \lambda)=\beta(\hat{x} \mid \lambda)$,

\footnotetext{
${ }^{18}$ For all $Q=\left(Q_{1}, Q_{2}, \cdots, Q_{\tau n}\right)$ with $-k_{i} \leq Q_{i} \leq k_{0}-k_{i}$ for all $i, \lim _{\tau \rightarrow \infty} \widetilde{W}_{\lambda}\left(Q_{1}, \cdots, Q_{\tau n}\right)$ is well-defined, because $\left\{\widetilde{W}_{\lambda}\left(Q_{1}, \cdots, Q_{\tau n}\right)\right\}_{\tau=1}^{\infty}$ is a Cauchy series and must converge to a finite number. Similarly, $\lim _{\tau \rightarrow \infty} \frac{1}{\tau} E\left[\sum_{i=1}^{\tau n} Q_{i}\left(v_{i}\right)\right]$ exists.
} 
and let $\hat{q}$ be the allocation defined as

$$
\widehat{q}_{i}(v)=\widehat{Q}_{i}\left(v_{i}\right)= \begin{cases}k_{0}-k_{i}, & \text { if } v_{i}>\hat{y} \\ 0, & \text { if } \hat{x} \leq v_{i} \leq \hat{y} \\ -k_{i}, & \text { if } v_{i}<\hat{x}\end{cases}
$$

One can verify that $E\left[\sum_{i=1}^{n} \hat{q}_{i}(v)\right]=E\left[\sum_{i=1}^{n} \widehat{Q}_{i}\left(v_{i}\right)\right]=0$. That is, the allocation $\hat{q}$ balances in expected terms; however it does not for all values of $v$. The allocation $\hat{q}$ corresponds to a simple bid-ask mechanism where the market maker sets two prices $\hat{x}$ and $\hat{y}$. A participant will sell all his units if and only if he is willing to sell them at a price equal to $\hat{x}$. Conversely, he will buy $\left(k_{0}-k_{i}\right)$ units if and only if he is willing to pay $\hat{y}$.

Theorem 6. As the number of replication of the economy increases, the optimal trading mechanism converges to the bid-ask mechanism $\hat{q}$. More precisely, let $q^{* \tau}$ be the optimal allocation given some $\tau$ and $\lambda$. Then for all $i$ and almost all $v_{i} \in[\underline{v}, \bar{v}]$, we have $\lim _{\tau \rightarrow \infty} Q_{i}^{* \tau}\left(v_{i}\right)=\widehat{Q}_{i}\left(v_{i}\right)$

Proof: We first compare the values for the objective function with allocations $\hat{q}$ and $q^{* \tau}$. We have

$$
\widetilde{W}_{\lambda}(\widehat{Q})-\widetilde{W}_{\lambda}\left(Q^{* \tau}\right)=\frac{1}{\tau} E\left[\sum_{i=1}^{n \tau}\left(\delta\left(v_{i} \mid \hat{x}, \lambda\right)-\mu\right) \widehat{Q}_{i}\left(v_{i}\right)-\left(\delta\left(v_{i} \mid x_{i}^{* \tau}, \lambda\right)-\mu\right) Q_{i}^{* \tau}\left(v_{i}\right)\right]
$$

Note that adding the constant $\mu$ has no effect on this differential since $E\left[\sum_{i=1}^{n \tau} Q_{i}^{* \tau}\left(v_{i}\right)\right]=E\left[\sum_{i=1}^{n \tau} \widehat{Q}_{i}\left(v_{i}\right)\right]=$ 0 . So if we let $\mu=\delta(\hat{x} \mid \hat{x}, \lambda)$, the above expression can be rewritten as

$$
\begin{aligned}
\widetilde{W}_{\lambda}(\widehat{Q})-\widetilde{W}_{\lambda}\left(Q^{* \tau}\right)= & \frac{1}{\tau} E\left[\sum_{i=1}^{n \tau}\left(\delta\left(v_{i} \mid \hat{x}, \lambda\right)-\delta(\hat{x} \mid \hat{x}, \lambda)\right)\left(\widehat{Q}_{i}\left(v_{i}\right)-Q_{i}^{* \tau}\left(v_{i}\right)\right)\right] \\
& +\frac{1}{\tau} E\left[\sum_{i=1}^{n \tau}\left(\delta\left(v_{i} \mid \hat{x}, \lambda\right)-\delta\left(v_{i} \mid x_{i}^{* \tau}, \lambda\right)\right) Q_{i}^{* \tau}\left(v_{i}\right)\right]
\end{aligned}
$$

This differential is positive because the expression in each expectation is positive. Indeed, when $v_{i}<\hat{x}, \delta\left(v_{i} \mid \hat{x}, \lambda\right)-\delta(\hat{x} \mid \hat{x}, \lambda) \leq 0$ and $\widehat{Q}_{i}\left(v_{i}\right)-Q_{i}^{* \tau}\left(v_{i}\right)=-k_{i}-Q_{i}^{* \tau}\left(v_{i}\right) \leq 0 ;$ when $\hat{x} \leq v_{i} \leq \hat{y}$, $\delta\left(v_{i} \mid \hat{x}, \lambda\right)-\delta(\hat{x} \mid \hat{x}, \lambda)=0$; when $v_{i}>\hat{y}, \delta\left(v_{i} \mid \hat{x}, \lambda\right)-\delta(\hat{x} \mid \hat{x}, \lambda) \geq 0$ and $\widehat{Q}_{i}\left(v_{i}\right)-Q_{i}^{* \tau}\left(v_{i}\right)=k_{0}-$ $k_{i}-Q_{i}^{* \tau}\left(v_{i}\right) \geq 0$. Hence $\left(\delta\left(v_{i} \mid \hat{x}, \lambda\right)-\delta(\hat{x} \mid \hat{x}, \lambda)\right)\left(\widehat{Q}\left(v_{i}\right)-Q_{i}^{* \tau}\left(v_{i}\right)\right) \geq 0$. Similarly, $\left(\delta\left(v_{i} \mid \hat{x}, \lambda\right)-\right.$ $\left.\delta\left(v_{i} \mid x_{i}^{*}, \lambda\right)\right) Q_{i}^{* \tau}\left(v_{i}\right) \geq 0$. When $Q_{i}^{* \tau}\left(v_{i}\right)>0, \delta\left(v_{i} \mid x_{i}^{* \tau}, \lambda\right)=\alpha\left(v_{i} \mid \lambda\right) \leq \delta\left(v_{i} \mid \hat{x}, \lambda\right)$ and when $Q_{i}^{* \tau}\left(v_{i}\right)<0$, $\delta\left(v_{i} \mid x_{i}^{* \tau}, \lambda\right)=\beta\left(v_{i} \mid \lambda\right) \geq \delta\left(v_{i} \mid \hat{x}, \lambda\right)$. The above implies that the simple bid-ask mechanism $\widehat{Q}$ dominates all the incentive-feasible mechanisms. 
Now consider an alternative allocation $\tilde{q}$ given by

$$
\tilde{q}_{i}\left(v_{i}, v_{-i}\right)= \begin{cases}\left(k_{0}-k_{i}\right) \min \left(1, \frac{\sum_{i \in N_{s}} k_{i}}{\sum_{i \in N_{b}}\left(k_{0}-k_{i}\right)}\right), & \text { if } v_{i}>\hat{y} \\ 0, & \text { if } \hat{x} \leq v_{i} \leq \hat{y} \\ -k_{i} \min \left(1, \frac{\sum_{i \in N_{b}}\left(k_{0}-k_{i}\right)}{\sum_{i \in N_{s}} k_{i}}\right), & \text { if } v_{i}<\hat{x} .\end{cases}
$$

where $N_{b}=\left\{i \in \tau N \mid v_{i}>\hat{y}\right\}$ and $N_{s}=\left\{i \in \tau N \mid v_{i}<\hat{x}\right\}$. The allocation $\tilde{q}$ resembles the allocation $\hat{q}$, however it is constructed to insure that resources balance ex post: $\sum_{i=1}^{n \tau} \tilde{q}(v)=0$ for all $v$. Since $q^{* \tau}$ maximizes $W_{\lambda}(\cdot)$ over all implementable and resource balancing allocations which include $\tilde{q}$, we must have $\widetilde{W}_{\lambda}\left(q^{* \tau}\right) \geq \widetilde{W}_{\lambda}(\tilde{q})$. Since $V^{*}\left(\widetilde{Q}_{i}\right)=V^{*}\left(\widehat{Q}_{i}\right)=[\hat{x}, \hat{y}]$, it follows that

$$
\widetilde{W}_{\lambda}(\widehat{Q})-\widetilde{W}_{\lambda}(\widetilde{Q})=\frac{1}{\tau} E\left[\sum_{i=1}^{n \tau}\left(\delta\left(v_{i} \mid \hat{x}, \lambda\right)-\delta(\hat{x} \mid \hat{x}, \lambda)\right)\left(\widehat{Q}_{i}\left(v_{i}\right)-\widetilde{Q}_{i}\left(v_{i}\right)\right)\right] \geq \widetilde{W}_{\lambda}(\widehat{Q})-\widetilde{W}_{\lambda}\left(Q^{* \tau}\right) .
$$

The main idea of the proof is to show that as the economy replicates, $\widetilde{Q}_{i}\left(v_{i}\right)$ converges to $\widehat{Q}_{i}\left(v_{i}\right)$ for all $i$ and $v_{i}$. Indeed, from the law of large numbers, the proportion of traders with an endowment of $l$ units and valuations greater than $\hat{y}$ equals $\frac{n_{l}}{n}(1-F(\hat{y}))$ when the market size is sufficiently large. Thus, the total demand for the good is

$$
\sum_{i \in N_{b}}\left(k_{0}-k_{i}\right)=\tau n \sum_{l=0}^{k_{0}}\left(k_{0}-l\right) \frac{n_{l}}{n}(1-F(\hat{y}))=\tau\left(k_{0} n-K\right)(1-F(\hat{y})) .
$$

In the same way, the proportion of traders with $l$ units and valuations less than $\hat{x}$ equals $\frac{n_{l}}{n} F(\hat{x})$, and the total supply is

$$
\sum_{i \in N_{s}} k_{i}=\tau n \sum_{l=0}^{k_{0}} l \frac{n_{l}}{n} F(\hat{x})=\tau K F(\hat{x})
$$

Hence we obtain

$$
\lim _{\tau \rightarrow \infty} \frac{\sum_{i \in N_{b}}\left(k_{0}-k_{i}\right)}{\sum_{i \in N_{s}} k_{i}}=\frac{\left(k_{0} n-K\right)(1-F(\hat{y}))}{K F(\hat{x})}=1
$$

with probability one, which implies that $\tilde{q}$ as defined in (20) converges to $\hat{q}$ and $\tilde{Q}$ converges to $\widehat{Q}$ when the market size increases.

It follows that $\widetilde{W}_{\lambda}(\widehat{Q})-\widetilde{W}_{\lambda}\left(Q^{* \tau}\right)$ converge toward zero. Hence from (19), for all $i$ and almost all $v_{i}$ we must have that $\left(\delta\left(v_{i} \mid \hat{x}, \lambda\right)-\delta(\hat{x} \mid \hat{x}, \lambda)\right)\left(\widehat{Q}\left(v_{i}\right)-Q_{i}^{* \tau}\left(v_{i}\right)\right)$ and $\left(\delta\left(v_{i} \mid \hat{x}, \lambda\right)-\delta\left(v_{i} \mid x_{i}^{* \tau}, \lambda\right)\right) Q_{i}^{* \tau}\left(v_{i}\right)$ converge to 0 as $\tau$ increases. If $\delta\left(v_{i} \mid \hat{x}, \lambda\right) \neq \delta(\hat{x} \mid \hat{x}, \lambda)$, i.e. when $v_{i}<\hat{x}$ or $v_{i}>\hat{y}$, from the first convergence we must have $Q_{i}^{* \tau}\left(v_{i}\right) \rightarrow \widehat{Q}\left(v_{i}\right)$. Now from the second convergence, either $Q_{i}^{* \tau}\left(v_{i}\right) \rightarrow 0$ or $\delta\left(v_{i} \mid x_{i}^{* \tau}, \lambda\right) \rightarrow \delta(h a t x \mid \hat{x}, \lambda)$ for all $v_{i} \in[\hat{x}, \hat{y}]$ (for which $\widehat{Q}\left(v_{i}\right)=0$ ), which implies that $x_{i}^{* \tau} \rightarrow \hat{x}$ 
and $y_{i}^{* \tau} \rightarrow \hat{y}$ which in turn implies that $Q_{i}^{* \tau}\left(v_{i}\right) \rightarrow 0$. In both cases, we have established that $Q_{i}^{* \tau}\left(v_{i}\right) \rightarrow \widehat{Q}_{i}\left(v_{i}\right)$.

Q.E.D

As the number of participants increases, the bid-ask price mechanism becomes more or less ex post resource balancing. So a mechanism relatively close to it may be used by the market-maker to maximize his objective function. Also as a corollary of Theorems 5 and 7 , we can show that ex ante efficiency converges to ex post efficiency as the market size increases (also see Gresik and Satterthwaite [3]).

Theorem 8. The ex ante efficient allocation coincides with the ex post efficient allocation, and can be implemented by setting a single price $\hat{x}=\hat{y}=F^{-1}(1-p)$ at which traders can buy or sell units as the market size increases.

Proof: Whenever $\lambda>0$, we have that $\hat{x}<\hat{y}$. So the bid-ask price mechanism $\hat{q}$ will generate strict positive revenue to the market-maker. As the market size increase, the optimal allocation $q^{* \tau}$ given $\lambda$ will converge to $\hat{q}$ and will also generate profits to the market-maker. So as the market size increases, we can decrease $\lambda$ towards zero and the solution to problem $\mathcal{P}_{s}$ converges to the ex post efficient allocation.

Q.E.D 


\section{Appendix}

Proof of Lemma 1. If the trading mechanism $\{q, t\}$ is incentive compatible, then for any two valuations $v_{i}, v_{i}^{*} \in[\underline{v}, \bar{v}]$,

$$
U_{i}\left(v_{i}\right)=v_{i} Q_{i}\left(v_{i}\right)-T_{i}\left(v_{i}\right) \geq v_{i} Q_{i}\left(v_{i}^{*}\right)-T_{i}\left(v_{i}^{*}\right)
$$

and

$$
U_{i}\left(v_{i}^{*}\right)=v_{i}^{*} Q_{i}\left(v_{i}^{*}\right)-T_{i}\left(v_{i}^{*}\right) \geq v_{i}^{*} Q_{i}\left(v_{i}\right)-T_{i}\left(v_{i}\right)
$$

These two inequalities imply that

$$
\left(v_{i}-v_{i}^{*}\right) Q_{i}\left(v_{i}\right) \geq U_{i}\left(v_{i}\right)-U_{i}\left(v_{i}^{*}\right) \geq\left(v_{i}-v_{i}^{*}\right) Q_{i}\left(v_{i}^{*}\right)
$$

Thus, if $v_{i}>v_{i}^{*}$, we must have $Q_{i}\left(v_{i}\right) \geq Q_{i}\left(v_{i}^{*}\right)$, so $Q_{i}\left(v_{i}\right)$ is non-decreasing. Furthermore, the above inequalities also imply that $U_{i}\left(v_{i}\right)$ is absolutely continuous, thus differentiable almost everywhere with derivative $\frac{d U_{i}}{d v_{i}}\left(v_{i}\right)=Q_{i}\left(v_{i}\right)$; or in the more convenient integral form

$$
U_{i}\left(v_{i}\right)=U_{i}\left(v_{i}^{*}\right)+\int_{v_{i}^{*}}^{v_{i}} Q_{i}(u) d u
$$

Now if $v_{i}^{*} \in V^{*}\left(Q_{i}\right)$, then $Q_{i}\left(v_{i}\right) \geq 0(\leq 0)$ for $v_{i}>v_{i}^{*}\left(<v_{i}^{*}\right)$, which imply that $U_{i}\left(v_{i}\right)-U_{i}\left(v_{i}^{*}\right)=$

$\int_{v_{i}^{*}}^{v_{i}} Q_{i}(u) d u \geq 0$ for all $v_{i}$; thus the expected net utility $U_{i}\left(v_{i}\right)$ is minimized at $v_{i}^{*}$ and $\{q, t\}$ is individually rational if and only if $U_{i}\left(v_{i}^{*}\right) \geq 0$.

Suppose now that the mechanism $\{q, t\}$ is such that $Q_{i}\left(v_{i}\right)$ is non-decreasing and $U_{i}\left(v_{i}\right)$ satisfies (18) for some $v_{i}^{*} \in V^{*}\left(Q_{i}\right)$ with $U_{i}\left(v_{i}^{*}\right) \geq 0$, then for any $v_{i}, \hat{v}_{i} \in[\underline{v}, \bar{v}]$,

$$
U_{i}\left(v_{i}\right)-U_{i}\left(\hat{v}_{i}\right)=\int_{\hat{v}_{i}}^{v_{i}} Q_{i}(u) d u \geq\left(v_{i}-\hat{v}_{i}\right) Q_{i}\left(\hat{v}_{i}\right)
$$

where the inequality follows from the fact that $Q_{i}(u)$ is non-decreasing in $u$. This inequality can be rewritten as

$$
U_{i}\left(v_{i}\right) \geq U_{i}\left(\hat{v}_{i}\right)+\left(v_{i}-\hat{v}_{i}\right) Q_{i}\left(\hat{v}_{i}\right)=v_{i} Q_{i}\left(\hat{v}_{i}\right)-T_{i}\left(\hat{v}_{i}\right)
$$

Thus, $\{q, t\}$ is incentive compatible. In the above, we have already shown that if $U_{i}\left(v_{i}^{*}\right) \geq 0$, an incentive compatible mechanism is also individually rational.

Q.E.D 
Proof of Lemma 2. For any function $q(v)$ such that $Q_{i}\left(v_{i}\right)$ is non-decreasing, if $\{q, t\}$ is an incentive feasible mechanism, from Lemma 1 the expected revenue of the mechanism equals

$$
\begin{aligned}
R & =E\left[\sum_{i=1}^{n} t_{i}(v)\right] \\
& =E\left[\sum_{i=1}^{n}\left(v_{i} Q_{i}\left(v_{i}\right)-U_{i}\left(v_{i}\right)\right)\right] \\
& =E\left[\sum_{i=1}^{n}\left(v_{i} Q_{i}\left(v_{i}\right)-\int_{v_{i}^{*}}^{v_{i}} Q_{i}(u) d u\right)\right]-\sum_{i=1}^{n} U_{i}\left(v_{i}^{*}\right)
\end{aligned}
$$

Integrating the first term on the right by parts, we obtain

$$
R=\sum_{i=1}^{n} E\left[\eta\left(v_{i} \mid v_{i}^{*}\right) Q_{i}\left(v_{i}\right)\right]-\sum_{i=1}^{n} U_{i}\left(v_{i}^{*}\right)
$$

Thus, the maximum expected revenue from any incentive feasible mechanism implementing $q(v)$ cannot be greater than

$$
R(q)=\sum_{i=1}^{n} E\left[\eta\left(v_{i} \mid v_{i}^{*}\right) Q_{i}\left(v_{i}\right)\right]
$$

To complete the proof, we must construct a payment function $t(v)$ so that $\{q, t\}$ is an incentive feasible mechanism leading $R(q)$. There are many such functions which could be used; we will consider a function defined as follows:

$$
t_{i}(v)=v_{i} q_{i}\left(v_{i}, v_{-i}\right)-\int_{v_{i}^{*}}^{v_{i}} q_{i}\left(u, v_{-i}\right) d u
$$

where $v_{i}^{*} \in V^{*}\left(Q_{i}\right)$. Then $U_{i}\left(v_{i}^{*}\right)=0$ and $U_{i}\left(v_{i}\right)=\int_{v_{i}^{*}}^{v_{i}} Q_{i}(u) d u$. From Lemma 1 , the mechanism $\{q, t\}$ is incentive feasible and has an expected revenue equal to $R(q)$. Thus, our proof of Lemma 2 is complete.

Q.E.D

Proof of Lemma 4. Randomization rules matter only when a group of participants share the same $x^{*}$. So let $x_{l}^{*}=x_{S}^{*} \forall l \in S=\{i, \cdots, j\}$ with $i \leq j$ and $x_{l}^{*} \neq x_{S}^{*} \forall l \notin S$. From Lemma 3, we have $\sum_{l=i}^{m} \widetilde{Q}_{l}\left(x_{S}^{*}\right) \leq 0$ for all $i \leq m<j$ and $\sum_{l=i}^{j} \widetilde{Q}_{l}\left(x_{S}^{*}\right)=0$. Typically, participants in $S$ with the lowest indexes will, according to the allocation rule $\tilde{q}$, have negative expected net trade. Hence, we need to increase the probability that the low index participants are awarded units in case of a tie to garantee that the expected net trades are equal for all participants in $S$.

We implement some randomization rule, by randomly assigning a hierarchy rank to each participant in the subset $S$. The tie breaking rule of $\tilde{q}$ is then used and gives participants assigned 
to higher hierarchy rank (instead of those with higher index) the advantage in case of a tie. The random assignment process is constructed in such a way that participants in subset $S$ have the same expected net trade, that is 0 . Note that when participant with index $m$ is given the rank $l$, his expected net trade, denoted by $\widetilde{Q}_{m}^{l}\left(x_{S}^{*}\right)$, is given by $\widetilde{Q}_{l}\left(x_{S}^{*}\right)+k_{l}-k_{m} \cdot{ }^{19}$ Thus for all $m$, we must have: $\sum_{l \in S} \alpha_{m}^{l}\left(\widetilde{Q}_{l}\left(x_{l}^{*}\right)+k_{l}\right)=k_{m}$, where $\alpha_{m}^{l}$ is the probability that participant with index $m$ is assigned rank $l$ in the hierarchy. To achieve this, one can construct a sequence of at most $(j-i-1)$ one-by-one random permutations from the initial index to a final hierarchy.

Let $m$ be the lowest index in $S$ with strictly negative net trade and $l$ be the lowest index in $S$ with strict positive net trade. We must have $m<l$, because otherwise we would have $Q_{h}\left(x_{S}^{*}\right)=0$ for $i \leq h \leq l-1$ and thus $\sum_{h=i}^{l} \widetilde{Q}_{h}\left(x_{S}^{*}\right)>0$. We assign some probability $\alpha_{m}^{l}$ that participant $m$ is given rank $l$ and vice versa. Given this random permutation between $m$ and $l$, the expected net trade for $m$ becomes $\left(1-\alpha_{m}^{l}\right) \widetilde{Q}_{m}\left(x_{S}^{*}\right)+\alpha_{m}^{l}\left[\widetilde{Q}_{l}\left(x_{S}^{*}\right)+k_{l}-k_{m}\right]$. Since $\widetilde{Q}_{m}^{l}\left(x_{S}^{*}\right)=\widetilde{Q}_{l}\left(x_{S}^{*}\right)+k_{l}-k_{m} \geq \widetilde{Q}_{l}\left(x_{S}^{*}\right)>0$ and $\widetilde{Q}_{m}\left(x_{S}^{*}\right)<0$, there exists a unique $\alpha_{m}^{l}$ such that the expected net trade of $m$ becomes zero. So we set $\alpha_{m}^{l}$ to the value for $m$ 's net expected trade to become zero. We next proceed with a new pair of participans $m$ and $l$, selected as before, given the new adjusted $Q_{i}^{\prime}$ s. In each round, we bring the expected net trade of at least one participant to zero, so after at most $(j-i-1)$ rounds, the expected net trades of all participants in $S$ will be zero.

Q.E.D

Proof of Lemma 5. For any function $q(v)$ such that $Q_{i}\left(v_{i}\right)$ is non-decreasing, if there exists a payment function $t$ so that $\{q, t\}$ is incentive feasible and $\sum_{i=1}^{n} t_{i}(v)=0$ for all $v$, then from Lemma 2, we must have $E\left[\sum_{i=1}^{n} \eta\left(v_{i} \mid v_{i}^{*}\right) q_{i}(v)\right] \geq 0$ where $v_{i}^{*} \in V^{*}\left(Q_{i}\right)$.

Suppose now that $q$ satisfies the above inequality, we must construct the payment function $t(v)$ so that $\{q, t\}$ is incentive feasible and $\sum_{i=1}^{n} t_{i}(v)=0$ for all $v$. Let

$$
c_{i}=\frac{1}{n} E\left[\sum_{i=1}^{n} \eta\left(v_{i} \mid v_{i}^{*}\right) q_{i}(v)\right]-\int_{\underline{v}}^{v_{i}^{*}} Q_{i}(u) d u-\frac{1}{n-1} \sum_{j \neq i} \int_{\underline{v}}^{\bar{v}}[u f(u)+F(u)-1] Q_{j}(u) d u
$$

\footnotetext{
${ }^{19}$ Since the traders' valuations are independently and identically distributed, we have

$\widetilde{Q}_{m}^{l}\left(x_{S}^{*}\right)+k_{m}=k_{0} \operatorname{Pr}\left\{\right.$ there are less than $n_{0}$ traders other than $m$ who have either valuation greater than $y_{S}^{*}$ and index lower than $l$ or valuation greater than $x_{S}^{*}$ and index higher than $\left.l\right\}$ $+r \operatorname{Pr}\left\{\right.$ there are exactly $n_{0}$ traders other than $m$ who have either valuation greater than $y_{S}^{*}$ and index lower than $l$ or valuation greater than $x_{S}^{*}$ and index higher than $\left.l\right\}$ $=\widetilde{Q}_{l}\left(x_{S}^{*}\right)+k_{l}$
} 
then a little algebra shows that $\sum_{i=1}^{n} c_{i}=0$. Hence let

$$
t_{i}(v)=v_{i} Q_{i}\left(v_{i}\right)-\int_{\underline{v}}^{v_{i}} Q_{i}(u) d u-\frac{1}{n-1} \sum_{j \neq i}\left[v_{j} Q_{j}\left(v_{j}\right)-\int_{\underline{v}}^{v_{j}} Q_{j}(u) d u\right]-c_{i},
$$

we have $\sum_{i=1}^{n} t_{i}(v)=0$. To complete the proof, it suffices to show that $\{q, t\}$ is incentive feasible.

By integration by parts, we obtain

$$
\begin{aligned}
T_{i}\left(v_{i}\right) & =v_{i} Q_{i}\left(v_{i}\right)-\int_{\underline{v}}^{v_{i}} Q_{i}(u) d u-\frac{1}{n-1} \sum_{j \neq i} \int_{\underline{v}}^{\bar{v}}[F(u)+u f(u)-1] Q_{j}(u) d u-c_{i} \\
& =v_{i} Q_{i}\left(v_{i}\right)-\int_{v_{i}^{*}}^{v_{i}} Q_{i}(u) d u-\frac{1}{n} E\left[\sum_{i=1}^{n} \eta\left(v_{i} \mid v_{i}^{*}\right) q_{i}(v)\right]
\end{aligned}
$$

Hence

$$
U_{i}\left(v_{i}\right)=v_{i} Q_{i}\left(v_{i}\right)-T_{i}\left(v_{i}\right)=\frac{1}{n} E\left[\sum_{i=1}^{n} \eta\left(v_{i} \mid v_{i}^{*}\right) q_{i}(v)\right]+\int_{v_{i}^{*}}^{v_{i}} Q_{i}(u) d u
$$

and in particular

$$
U_{i}\left(v_{i}^{*}\right)=\frac{1}{n} E\left[\sum_{i=1}^{n} \eta\left(v_{i} \mid v_{i}^{*}\right) q_{i}(v)\right] \geq 0
$$

so Lemma 1 guarantees that $\{q, t\}$ is incentive feasible.

Q.E.D 


\section{References}

[1] K. Chatterjee and W. Samuelson, Bargaining under incomplete information, Operations Research 31 (1983), 835-851.

[2] P. Cramton, R. Gibbons and P. Klemperer, Dissolving a partnership efficiently, Econometrica 55 (1987), 615-632.

[3] T. Gresik and M. Satterthwaite, The rate at which a simple market becomes efficient as the number of traders increases: An asymptotic result for optimal trading mechanisms, J. Econ. Theory 48 (1989), 304-332.

[4] B. Holmström and R. B. Myerson, Efficient and durable decision rules with incomplete information, Econometrica 51 (1983), 1799-1820.

[5] J.-J. Laffont and J. Tirole, "A Theory of Incentives in Procurement and Regulation", The MIT Press, 1993.

[6] J.-J. Laffont, E. Maskin and J.-C. Rochet, Optimal non-linear pricing with two dimensional characteristics, In "Information, Incentives, and Economic Mechanisms", ed. by T. Groves, R. Radner, and S. Reiter. University of Minnesota Press, 1987.

[7] W. Leininger, P. B. Linhart and R. Radner, Equilibria of the sealed-bid mechanism for bargaining with incomplete information, J. Econ. Theory 48 (1989), 63-106.

[8] H. Lu, Efficient Performance in a Simple Market with Incomplete Information, Université de Montréal, 1998.

[9] L. Makowski and C. Mezzetti, The possibility of efficient mechanisms for trading an indivisible object, J. Econ. Theory 59 (1993), 451-465.

[10] E. S. Maskin and J. G. Riley, Optimal multi-unit auctions, in The economics of missing markets, information and games, ed. by F. Hahn. Oxford: Oxford University Press, 1989.

[11] R. P. McAfee, Efficient allocation with continuous quantities, J. Econ. Theory 53 (1991), 51-74. 
[12] M. Mussa and S. Rosen, Monopoly and Product Quality, J. Econ. Theory 18 (1987), 301-317.

[13] R. B. Myerson, Incentive compatibility and the bargaining problem, Econometrica 47 (1979), $61-73$.

[14] R. B. Myerson, Optimal auction design, Math. Oper. Res. 6 (1981), 58-73.

[15] R. B. Myerson and M. Satterthwaite, Efficient mechanisms for bilateral trading, J. Econ. Theory 28 (1983), 265-281.

[16] J.-C. Rochet, Ironing, sweeping and multidimensional screening, mimeo (1995), Toulouse University.

[17] A. Rustichini, M. A. Satterthwaite, and S. R. Williams, Convergence to efficiency in a simple market with incomplete information, Econometrica 62 (1994), 1041-1063.

[18] M. Satterthwaite and S. R. Williams, Bilateral trade with the sealed-bid k-double auction: existence and efficiency, J. Econ. Theory 48 (1989), 107-133.

[19] D. Spulber, Bargaining and regulation with asymmetric information about demand and supply, J. Econ. Theory 44 (1988), 251-268.

[20] S. R. Williams, Efficient performance in two-agent bargaining, J. Econ. Theory 41 (1987), 154172.

[21] R. Wilson, Incentive efficiency of double auctions, Econometrica 53 (1985), 1101-1115.

[22] R. Wilson, Efficient trading, in Issues in contemporary microeconomics and welfare, ed. by G. Feiwel. London: The Macmillan Press Ltd (1985). 